\title{
LES SERVICES BANCAIRES ET D'ASSURANCES ET LES SERVICES INFORMATIQUES DANS LES VILLES MOYENNES DU GRAND SUD-OUEST FRANÇAIS, LOGIQUES DE DÉPLOIEMENT ET STRATÉGIES D'ANCRAGE
}

\author{
Jean-Marc Zuliani \\ Université Toulouse II - Le Mirail, LISST-CIEU \\ zuliani@univ-tlse2.fr
}

\begin{abstract}
Résumé : Dans les villes moyennes du Grand Sud-ouest français (Régions Aquitaine, Midi-Pyrénées et Languedoc-Roussillon) la distribution des services bancaires et d'assurances et des services informatiques s'opère selon des modalités distinctes. Si certains établissements bancaires et d'assurances à assimilent les villes moyennes à des points de contacts commerciaux, d'autres, parmi la sphère des groupes mutualistes, y déploient des fonctions décisionnelles. Un développement polarisé des services informatiques affecte plusieurs villes moyennes qui recèlent des sources de marchés et des creusets de compétences. Les villes moyennes s'intègrent dans des flux plus globaux faits d'une circulation de compétences et de savoirs spécialisés, via le déploiement et l'activité sur leur territoire de prestataires de services informatiques. Dans le Grand Sud-ouest français, la diffusion territoriale des services financiers et informatiques jette les bases d'un nouveau "système de villes moyennes".
\end{abstract}

Most-Clés : Grand Sud-ouest français, services bancaires, services informatiques, système urbain.

Resumen: En el suroeste de Francia (regiones de Aquitania, MediodíaPirineos y Laguedoc-Rosellón) se está cimentando un nuevo sistema de ciudades medianas sobre los servicios financieros (banca y aseguradoras) e informáticos con modelos de implantación espacial diferente. Mientras que algunos establecimientos bancarios consideran a las ciudades intermedias simples espacios de operaciones comerciales, las mutualidades desarro-

Recibido: 24-04-13. Aceptado: 25-11-13. 
llan en ellas funciones que conllevan tomas de decisiones estratégicas. Por otra parte, los servicios informáticos se polarizan en estos núcleos urbanos para luchar por un mercado cada vez más competitivo y global, apoyándose en las ciudades intermedias que favorecen los servicios, redes y recursos humanos.

Palabras clave: suroeste fancés, servicios financieros, servicios informáticos, sistema urbano.

\section{Introduction}

Les villes moyennes, tels que de nombreuses travaux l'ont souligné (Brunet, 1997 ; Saint-Julien, 2003 ; Rozemblat, Cicille, 2003 ; Méndez et alii.; 2010 ; Santamaria, 2012), conservent par tradition une fonction d'intermédiation entre le monde urbain métropolitain et l'espace rural, lui-même plus ou moins maillé par ses bourgs et petites villes. Au-delà de cette vision christallérienne classique de l'organisation des armatures urbaines, les villes dites " moyennes ", sans qu'il n'existe une définition établie ni un seuil numérique reconnu, offrent des profils économique et urbain très hétérogènes. La position supposée " intermédiaire " des villes moyennes ainsi que leur caractère disparate nourrissent des interrogations quant à l'impact et au rôle des activités de services marchands qui s'y déploient. Aujourd'hui, si les activités de services comptent pour plus des deux tiers des richesses créées dans les territoires des pays $\mathrm{OCDE}^{1}$, leur répartition spatiale s'opère en premier lieu au profit des métropoles régionales ou des villes de rang métropolitain (Mouhoud, 2010). Par nature, ces cités recèlent une masse critique d'externalités et conditions économiques propices à l'essor des services marchands comme la densité des échanges et des marchés de consommation, ou encore le caractère dense et diversifié des compétences circulantes parmi les marchés du travail locaux. Ces facteurs contribuent à faire des services à la production notamment, une base économique prépondérante des espaces métropolitains (Gallouj et alii., 2006). Mais face à la banalisation des services marchands, tant aux particuliers qu'aux entreprises, parmi les diverses strates du réseau urbain, l'un des enjeux est de cerner leur représentation, leur spécificité et leur comportement dans l'armature des villes moyennes que l'on circonscrira à un espace interrégional d'étude, celui du Grand Sud-ouest français (Aquitaine, Midi-Pyrénées et Languedoc-Roussillon) ${ }^{2}$.

1. Rapport 2005 de l'OCDE " Les services et la croissance économique ", 26 p.

2. Cette communication procède d'un travail contractuel de recherche (2007-2010) subventionné par le Ministère français de l'Écologie et de l'Aménagement Durable (cellule " Plan Urbain Construction Architecture "), et intitulé "Les services aux particuliers et aux entreprises, vecteurs d'un fonctionnement en réseau des villes moyennes ? L'exemple du Grand Sud-ouest français " (Jean-Marc Zuliani), 372 p., 2010. 


\section{Le rôle des villes moyennes dans le déploiement des services bancaires, d'assurances et informatiques}

\subsection{Une classification des villes moyennes en trois catégories principales d'espaces urbains}

À l'instar du reste du territoire français, les villes moyennes du Grand Sud-ouest français se situent dans un spectre assez large en termes d'habitants pour les agglomérations urbaines concernées (de 20000 à 200000 / 250 000) (Figure 3). Or, la dimension plutôt large de cette classe démographique renvoie un découpage officiel quelque peu imprécis, établi pourtant de longue date en France par la DATAR dans les années 1970. Même si cette catégorisation apparaît contestée, comme l'atteste l'existence d'une " fédération nationale des villes moyennes ", lobby territorial et institutionnel des agglomérations urbaines de 20000 à... 100000 habitants, son acception tacite conduit à recenser parmi la catégorie "villes moyennes " des profils de cités très distinctes tant sur le plan administratif, institutionnel qu'économique. Comme le souligne F. Taulelle (2010), la caractérisation par les fonctions urbaines apparât pertinente pour établir une classification des villes moyennes, et la taille joue un rôle discriminant : plus la ville est importante sur le plan démographique et des emplois, plus elle conforte ses fonctions urbaines. A l'inverse, plus la ville moyenne est de faible importance dans le registre démographique et des emplois, plus ses fonctions demeurent limitées. L'influence de la ville moyenne est dépendante de sa taille et de ses fonctions dans le registre des services supérieurs publics par exemple (fonction préfectorale, hospitalière, universitaire, services financiers et bancaires... etc) bien que l'on puisse s'interroger également sur l'influence territoriale que développerait à différentes échelles (locale, régionale ou nationale) la concentration locale de services spécifiques rendus aux entreprises (informatique, ingénierie ou logistique-transports).

Dans le spectre large de 20000 et 250000 habitants pour les agglomérations urbaines, les espaces des villes moyennes dans le Grand Sud-ouest français tendent à répartir en trois grandes catégories :

- D'abord, une catégorie de " grandes " villes moyennes dont les communes-centres oscillent entre 100000 et 200000 habitants (Perpignan, Nîmes) qui constituent avec leur agglomération, des pôles " proto-métropolitains " par leur statut préfectoral et par la concentration des fonctions décisionnelles d'intérêt infrarégional dans des services publics supérieurs (universités de plein exercice et centres de recherche; fonction hospitalière de type $\mathrm{CHU}$ ), des fonctions de siège intermédiaire pour les entreprises de réseau (La Poste, EDF, Orange-France Télécom entre autres), pour des grands enseignes commerciales, des prestataires de services aux entreprises (opérationnels ou de haute technologie), sans compter la structuration locale de filières dans des registres d'activités divers (logistique-transports, conditionnement des denrées agricoles et production agro-alimentaire, biotechnologies, services informatiques... etc). 
- Ensuite, une catégorie de villes moyennes dites " médianes " dotées de communescentres allant de 50000 à 100000 habitants (Pau, Tarbes, Béziers, Bayonne, Albi, Narbonne), certes bien inscrites dans leur département mais au statut administratif plus variable réunissant des villes préfectures et des sous-préfectures. Leur agglomération excède ou avoisine 100000 habitants avec une concentration moins importante de services publics supérieurs quand ils ne sont pas sous-représentés ou même parfois absents, et alors que se déploie une infrastructure moins dense de centres décisionnels intermédiaires pour les grandes entreprises des secteurs des télécommunications, de l'énergie ou du courrier même si elles peuvent être siège infrarégional de banques coopératives ou d'organismes d'assurances. Cette strate "intermédiaire " de villes moyennes se caractérise par la dimension émergente ou le renforcement de niches d'activités innovantes qui ont pu se structurer à la faveur d'une construction de compétences dans des domaines spécialisés de R\&D (Hydrogène à Albi, électronique de puissance à Tarbes, technologies d'exploration pétrolière et matériaux composites à Pau... etc) en lien avec l'implantation d'un institut public ou privé de recherche et la présence d'une grande firme industrielle génératrice d'un tissu connexe de sous-traitants dans l'usinage ou dans l'ingénierie.

- Enfin, un ensemble très composites de "petites " villes moyennes qui maillent les régions du Grand Sud-ouest français dont les communes-centres n'excèdent pas 50000 habitants pour des agglomérations inférieures à 80000 habitants (Agen, Castres, Alès, Rodez, Périgueux, Mont-de-Marsan...). Certes, les fonctions de production apparaitraient plutôt en retrait face à une économie résidentielle de consommation et au maintien des fonctions régaliennes en lien avec leur statut préfectoral ou sous-préfectoral dans leur département. Ces villes moyennes de rang mineur constituent des relais gestionnaires au déploiement territorial des services dans l'énergie, la banque et les assurances, la comptabilité, le négoce de détail ou en gros, alors que des trajectoires spécifiques ont conduit certains d'entre elles à développer grâce à des avantages comparatifs (bassin environnant de production agricole, établissements industriels aux fonctions de R\&D et de fabrication, institut public de recherche), une spécialisation dans des filières d'activités courantes (logistique-transports) ou innovantes (pharmaceutique, biotechnologies, mécanique de précision...).

Les trajectoires économique et démographique des villes moyennes du Grand Sudouest français offrent des contrastes marqués. Une fois de plus, il semble plus aisé d'interpréter le rôle et la fonction de ces villes au plan régional à l'aune de facteurs spécifiques que de causalités communes. Tout d'abord, les villes moyennes s'avèrent dans leur grande majorité le résultat d'une relation séculaire avec leur environnement infrarégional fait d'espaces ruraux et de villes plus petites. Le référent territorial est important et explique le maintien des fonctions de gestion, administration et décision parmi les différentes villes. Ce cadre territorial est souvent le département d'autant que ces villes moyennes dans leurs diversités sont souvent des préfectures ou des sous-préfectures : elles ont intégré à ce titre des attributs administratifs aux effets d'attraction sur les populations. Plusieurs d'entre elles (Albi, Alès, Nîmes) ont connu très tôt, parfois dès le XIXème siècle, un développement économique caractéristique de celui d'un 
bassin industriel (industries extractive, sidérurgie, textile) plutôt centré sur des tâches de mises en œuvre et d'exécution de la production. Cette logique de développement fordiste, fondée sur une division sociale des tâches, va par la suite s'étendre à d'autres villes dans la première partie du XXème siècle (Tarbes, Pau, Bayonne, Castres) à la faveur de mouvements de délocalisation industrielle, alors que plusieurs cités demeurent des pôles de centralités administratifs ou de services à la personne très pourvus en emplois publics (Carcassonne, Agen ou Rodez entre autres).

Les bases de développement de nombre de ces villes moyennes dans le Grand Sudouest français vont peu à peu évoluer à partir des années 70 et 80 mais selon des trajectoires diversifiées. Plusieurs villes autrefois structurées à partir d'une fonction de centre de production dans des activités industrielles vont affronter des processus de reconversion parfois douloureux qui se soldent par la disparition d'un patrimoine industriel qui avait assuré jusque là leur prospérité et des situations de plein emploi. D’autres villes, jusque là cantonnées à une fonction de centralité préfectorale et administrative, vont construire de nouvelles logiques de développement à la faveur de politiques publiques territoriales qui les dotent de nouveaux attributs dans les activités universitaires et la recherche (Perpignan) et qui sont parfois constitutives des processus de reconversion économique (Alès, Albi, Tarbes). Ailleurs, le développement des villes moyennes correspondra au renforcement d'une vocation spécialisée construite à partir du renforcement d'une activité industrielle dans un ou deux secteurs dominants (la mécanique automobile et l'agroalimentaire à Rodez, la pharmaceutique à Agen ou à Castres, la construction aéronautique à Pau ou à Tarbes, des services informatiques à Biarritz et à Nîmes), alors que dans d'autres cités, des initiatives autonomes des milieux locaux conduisent à créer des centres informatiques et techniques dédiés aux banques et assurances (Rodez, Bayonne, Albi, Agen). Pour des villes inscrites sur des axes majeurs de circulation à proximité de la frontière espagnole (Bayonne, Narbonne et Perpignan), s'affirme une fonction de " hub " logistique dans le transport du fret, le conditionnement et l'entreposage que renforce la proximité de bassins agricoles pour l'expédition des productions.

Ces trajectoires diverses soulignent l'impératif pour les villes moyennes de développer des stratégies spécifiques pour exister face aux espaces métropolitains régionaux (Bordeaux, Toulouse et Montpellier). D'où, la recherche dans nombre d'entre elles de stratégies de différenciation qui passent par l'affirmation de systèmes productifs centrés sur l'innovation de produits et de services à partir de relations de co-études entre un important établissement industriel, à l'implantation ancienne et versé dans la recherche et développement, et un tissu connexe de sous-traitants d'ingénierie de haut niveau (ex. du Centre Scientifique et Technique de Total à Pau ou des Laboratoires P. Fabre à Castres). Même si le poids des services aux entreprises demeure encore très discriminant entre les villes moyennes et les métropoles au sein du Grand Sud-ouest (Zuliani, 2004 ; Zuliani et Tallec, 2012), l'émergence de quelques unes de ces activités prestataires reste alors liée comme dans les services informatiques à la présence d'un secteur industriel, tertiaire ou administratif ou d'une activité économique, structurant la ville moyenne. 


\subsection{L'intérêt d'une approche centrée sur la distribution des services bancaires et des activités logicielles}

Parmi les régions Aquitaine, Midi-Pyrénées et Languedoc-Roussillon, les cités dites " moyennes " assurent un maillage territorial qui s'exerce de façon variable selon leur rang, leur importance démographique et leur influence en matière d'offre de services. En retenant deux catégories prépondérantes d'activités marchandes de services (les services bancaires et d'assurance ; les services informatiques), et en mettant l'accent sur leur organisation territoriale, la démarche choisie vise à caractériser le rôle et la fonction des villes moyennes selon la nature sectorielle des prestations étudiées. Les activités bancaires et d'assurances forment des branches représentatives de services marchands " obligés " et de proximité aussi bien pour les entreprises, les administrations que pour les particuliers. Leur déploiement territorial demeure un indicateur de dynamisme démographique d'un territoire. L'essor des services bancaires et d'assurances, considérés comme des services de consommation et de proximité, peut être interprété pour les villes moyennes comme l'indice d'un accroissement des logiques résidentielles et de peuplement. Aussi, les implantations corrélées des cellules bancaires d'expertise financière aux entreprises renforceraient un maillage territorial depuis les aires métropolitaines, et feraient des villes moyennes des espaces intermédiaires de décision. De leurs côtés, les services informatiques revêtent une grande diversité d'activités, des plus communs et standardisées (infogérance, technologies de l'information IT, gestion de production) à celles nécessitant une intensité forte de connaissances pour la conduite des projets comme ceux dédiés à la réalisation de systèmes d'information (ingénierie en informatique scientifique et technique). Ils ont aussi comme particularité d'entretenir une relation cognitive entre divers marchés de clientèles : le redéploiement des compétences et savoirs vers des marchés distincts forme un attribut de leur fonctionnement prestataire. Cette capacité à intervenir auprès de clientèles de branches d'activités différentes pour le développement de "technologies de l'information et de la communication" (TIC), fait des services informatiques un registre d'activités à la diffusion territoriale élargie, même si la densité territoriale des clientèles (industrielles et tertiaires) exerce un rôle discriminant à l'égard de leur distribution géographique.

La couverture des territoires de Midi-Pyrénées, d'Aquitaine et de Languedoc-Roussillon par les activités bancaires et d'assurances, conduit plusieurs villes moyennes-clés, indépendamment de leur taille et de leur localisation, à assumer des fonctions d'intermédiation gestionnaire. Le développement des services informatiques renvoie autant au développement de l'innovation parmi les entreprises qu'au renforcement des liens locaux avec des spécialités scientifiques liées aux TIC, sans compter les effets produits par une gouvernance locale qui valorise la compétitivité économique et l'appui à l'innovation dans les activités logicielles (Zuliani et Tallec, 2012). Dans quelle mesure, l'innovation dans les services informatiques participerait d'un processus territorialisé stimulé non seulement par des ressources localement ancrées mais aussi par un contexte social et institutionnel ? À ce stade, la ville moyenne incarnerait un espace dynamique 
en concentrant des relations entre les services informatiques et d'ingénierie, les marchés, les compétences, la culture technique et les représentations novatrices qu'elle génère comme espace d'innovation. Une conception systémique de l'innovation a progressivement a émergé : elle met l'emphase sur l'importance du territoire et le rôle de la proximité, notamment à travers les logiques d'interactions, pour assurer la création de connaissances et ressources nouvelles (Asheim et Gertler, 2005; Cooke et alii., 2004). Aussi, l'attention portée aux villes moyennes et à l'innovation dans les activités logicielles n'est pas étrangère à l'affirmation des concepts appliqués à l'innovation régionale. Les nombreuses recherches conduites par exemple sur les milieux innovateurs, les districts industriels ou encore les systèmes productifs locaux cherchent dans leur très grande majorité à appréhender les rapports qui s'instaurent entre innovation et territoire. A propos des villes moyennes, prévaut dès lors un double questionnement :

- Pourquoi dans un contexte donné, une ville moyenne connaît-elle une dynamique différente d'une autre concernant la genèse locale de services informatiques, d'ingénierie ou bien d'activités d'édition logicielle?

- Quelles sont les raisons pour lesquelles certaines villes moyennes plus que d'autres affirment dans ces registres sectoriels plus d'innovations?

Si des systèmes d'innovation se dessinent parmi des villes moyennes du moins dans les services associés aux TIC, l'idée selon laquelle la métropolisation les exclurait du champ des services de haut niveau aux entreprises conduit à un légitime réexamen (Baudelle, Tallec, 2008). Sans nier l'existence de disparités réelles entre les villes moyennes du Grand Sud-ouest, la consolidation d'une masse critique d'aménités urbaines et économiques dans plusieurs d'entre elles, contribuerait à atténuer les forts contrastes passés avec les espaces métropolitains (Bordeaux, Toulouse et Montpellier). D'où l'intérêt d'établir à l'échelle des villes moyennes, des corrélations entre le dynamisme des services " cognitifs" (services de conseil et d'ingénierie informatique, édition logicielle), les pratiques collaboratives des instituts académiques d'enseignement et de recherche dans les TIC, et la capacité innovante d'établissements industriels locaux adossés à des centres publics de recherche.

L'enjeu est à ce stade de distinguer au travers des services pris en compte, le fonctionnement en réseau de firmes qui les caractérisent. Dans le cas des services informatiques, la concentration d'entreprises établie à l'échelle de certaines villes moyennes intervient dans un environnement géographique marqué par la proximité, et dans lequel les firmes prestataires ou éditrices de software entretiennent des relations avec d'autres entreprises industrielles ou de services, des instituts scientifiques sachant que ces relations peuvent dépasser les seules logiques marchandes (échanges et circulations d'informations, de salariés et de compétences). L'organisation territoriale du réseau d'entreprises se fonde alors sur une proximité territoriale et une proximité organisée : les entreprises de services informatiques et d'édition logicielle s'inscrivent dans un espace 
local de relations à partir duquel se nouent des interactions avec d'autres acteurs industriels, scientifiques et de services, caractéristique d'un fonctionnement en système productif. Au contraire, l'évocation du terme "réseau " pour les banques et les assurances renvoie au déploiement d'activités de services qui reposent sur des réseaux de distribution et de diffusion des prestations diversifiées, fournies par les établissements bancaires (dépôt et gestion de comptes, épargne, prêts financiers, conseils et ventes de produits financiers divers) ou les compagnies d'assurances (négociations de contrats, conseils). Le déploiement de ces services en réseau parmi les armatures urbaines tient à la convergence dans les années 80 et 90 de l'informatique et des télécommunications, rendue possible par la technologie numérique, qui peut favoriser leur pilotage ou leur gestion centralisée ou décentralisée. Les services en réseau propres aux banques et aux compagnies d'assurances se caractérisent par la recherche d'économies d'échelle afin de couvrir par des structures de vente et de gestion les territoires pour diffuser leurs services en direction des clientèles.

\subsection{Le Grand Sud-ouest français, un maillage infrarégional vivace à partir des villes moyennes}

En dépit des recompositions qui font désormais de certaines villes moyennes des territoires orientés vers le développement de biens et services innovants (Tallec et Baudelle, 2008 ; Tallec 2102), les écarts avec les fonctions des espaces métropolitains régionaux demeurent marquants (Mouhoud, 2010). Le déséquilibre de l'armature urbaine n'est plus à démontrer dans le Grand Sud-ouest français : les capitales régionales (Bordeaux, Toulouse et à un degré moindre Montpellier) ont parfois tendance parfois a être qualifiées de " cathédrales dans le désert " : ce sont de grands chefs-lieux de régions vastes (Aquitaine, Midi-Pyrénées et Languedoc-Roussillon) qui concentrent une économie de services informatiques et d'ingénierie développée en particulier l'espace métropolitain toulousain (Scott et Zuliani, 2006). Ces positions de force métropolitaines s'avèrent rarement concurrencées, et demeurent l'apanage de Bordeaux, Toulouse et Montpellier, sur la base d'un héritage et d'un rayonnement renouvelés. Depuis les années 1990, ces métropoles ont consolidé leur suprématie en concentrant la plus grande partie de la croissance démographique de l'espace interrégional, et alors qu'à l'échelle de Midi-Pyrénées se dessinait une polarisation écrasante des emplois et activités de services, tant à la production qu'aux ménages, dans l'aire métropolitaine toulousaine (Zuliani, 2003).

Toulouse, Bordeaux et Montpellier ont à défendre un enjeu primordial qui consiste à conquérir une place dans la strate des métropoles régionales susceptibles de jouer un rôle aux niveaux national et même européen. Cet objectif les incite à capter pour elles-mêmes les activités de services supérieurs, publics ou marchands, les plus compétitives. Pourtant, on peut appréhender l'espace du Grand Sud-ouest autrement : les villes moyennes sont au cœur d'un quadrillage de l'espace interrégional essentiellement 
à partir de fonctions basiques préfectorales ou sous-préfectorales départementales bien que leur taille soit sans commune mesure avec celle des métropoles régionales. Dès lors, comment les activités de services bancaires et informatiques confortent, modifient ou bien remettent en cause le rôle des villes moyennes parmi le réseau urbain du Grand Sud-ouest? Cette interrogation se pose avec acuité au moment où les villes moyennes forment en quelque sorte un maillon "faible " des systèmes territoriaux, prises qu'elles sont entre le renforcement de la métropolisation et le développement démographique et économique de nouveaux espaces ruraux, lesquels ignorent par certains aspects les fonctions de centralité historique des villes moyennes (Béhar, 2004).

Appréhender les mutations autour des services informatiques, bancaires et d'assurances dans les villes moyennes requiert le traitement des données statistiques sur l'emploi salarié, basé sur des effets de seuil ou de masse critique. L'information statistique relative aux emplois salariés du secteur privé procédait jusqu'à l'année 2009 des données en ligne fournies l'UNEDIC, organisme français de gestion de l'assurance chômage, alors en charge via une structure-relais (l'ASSEDIC) du versement des indemnités de chômage. La constitution d'un " corpus " propre aux emplois dans les services informatiques pour la période 2001-2007 s'est fondée sur l'agrégation de plusieurs nomenclatures d'activités françaises (NAF) retenues parmi la nomenclature officielle en 700 postes de l'INSEE ${ }^{3}$. Or, à la suite de la réforme administrative de 2009 qui a engendré la fusion entre l'ASSEDIC et l'ANPE (structure de suivi et d'encadrement des personnes inscrites au chômage) puis la création de la structure "Pôle Emploi ", la gestion des informations statistiques en ligne sur l'emploi salarié a été décentralisée à l'échelle de chaque région ; chaque direction régionale de "Pôle Emploi " se réservant la décision de publier ou non ses résultats statistiques. De facto, la réactualisation des données après 2009 a été rendue difficile par l'absence d'une diffusion systématique des données sur l'emploi privé par branche d'activité. Dans le Grand Sud-ouest français, seule la région Languedoc-Roussillon propose à ce jour une mise en ligne actualisée sur son site "Pôle Emploi " des données statistiques sur l'emploi salarié. Dès 2008, l'INSEE a procédé en outre à l'édition d'une nouvelle grille de nomenclatures d'activités économiques, ce qui a obligé à une réactualisation des données à partir d'un nouveau " corpus " des services informatiques, fondé sur l'agrégation des nouvelles $\mathrm{NAF}^{4}$.

Les valeurs quantifiées relatives à l'évolution des emplois salariés dans les services informatiques, somme toute faibles en volume à l'échelle des villes considérées, justi-

3. Codes NAF mobilisés (jusqu'à 2007) : 721Z : Conseil en systèmes informatiques ; 722A : Editions de logiciels (non personnalisés) ; 722C : Autres activités de réalisation de logiciels ; 723Z : Traitement de données; $724 \mathrm{Z}$ : activités de banque de données ; 741C : Activités comptables ; 741E : Etudes de marché et sondages.

4. Codes NAF mobilisées (à partir de 2008) : 6201Z : Programmation informatique ; 6202A : Conseil en systèmes et logiciels informatiques ; 6202B : Tierce maintenance de systèmes et d'applications informatiques ; 6203Z Gestion d'installations informatiques; 6209Z: Autres activités informatiques ; 6311Z : Traitement de données, hébergement et activités connexes ; 6312Z : Portails Internet ; 6920Z : Activités comptables. 
fient le recours à une connaissance empirique des processus relationnels, constitutifs du développement de l'offre prestataire et de la construction des marchés de services. A partir d'entretiens, nous avons travaillé à la reconstitution des trajectoires des entreprises de services informatiques et à l'organisation en réseau des établissements bancaires et d'assurances. La méthode retenue a reposé sur la conduite entre 2008 et 2011 de 62 entretiens semi-directifs auprès de fondateurs, de responsables d'entreprises de services informatiques ou d'établissements bancaires ou d'assurances, complétés dans les villes moyennes étudiées, par des entrevues systématiques auprès d'acteurs institutionnels, de la recherche académique, du développement économique qui ont intégré les services informatiques, et plus généralement les services high-tech, ainsi que le maillage de leur territoire par les établissements bancaires et d'assurances, comme des variables structurantes du paysage économique local.

Pour les entreprises de software, le panel a été constitué sur la base du degré d'innovation en rapport avec l'offre de prestations ou de produits logiciels, en tenant également compte des liens de proximité avec les principaux établissements industriels ou tertiaires locaux ainsi que d'instituts de recherche. Une attention particulière a été portée aux reconstitutions des chaînes relationnelles, leur genèse et leurs évolutions qui permettent de saisir la construction géographique et sociale des marchés ou du moins leur ancrage dans un environnement social et institutionnel pourvoyeur de ressources (matérielles, financières, résiliaires et cognitives). Pour les activités bancaires et d'assurances, l'échantillon des établissements pris en compte a inclus les organismes de type coopératif (Crédit Agricole, Groupe Banques Populaires, Groupama), fondés sur un principe d'organisation et gestion des établissements à partir d'un sociétariat local, et les établissements de type de commercial (BNP, Société Général, HSBC, Generali... etc) contrôlés par un actionnariat boursier et déployés dans les régions via des sièges et des agences commerciales. Leur organisation géographique, appréhendée à partir de la répartition des fonctions entre les espaces métropolitains et les villes moyennes, a été au centre de nos préoccupations tout comme l'évolution de l'offre prestataire des banques en lien avec la demande des entreprises dans les villes moyennes. Les éléments structurels relatifs aux services informatiques et aux activités bancaires et d'assurances nous ont amenés à moduler les différentes échelles territoriales et les niveaux d'action dans lesquels évoluent les établissements prestataires, plus spécialement dans les zones d'emploi $^{5}$ de 12 villes retenues ${ }^{6}$, tant pour la diversité de leur localisation dans le Grand Sudouest que pour l'évolution variable des emplois.

5. Zone d'emploi : c'est un espace géographique déterminé par l'Institut National de la Statistique et des Etudes Economiques à l'intérieur duquel la plupart des actifs résident et travaillent, et dans lequel les établissements peuvent trouver l'essentiel de la main-d'œuvre pour occuper les emplois offerts.

6. Conurbation Bayonne-Biarritz, Pau, Tarbes, Agen, Montauban, Albi, Castres, Rodez, Perpignan, Narbonne, Béziers, Nîmes. 


\subsection{Une redistribution territoriale des services à partir de facteurs " génériques " communs}

L'affirmation d'une offre diversifiée de services conduit à l'évocation d'avantages compétitifs qu'offriraient les territoires des villes moyennes pour l'implantation d'entreprises de services informatiques et le déploiement d'établissements bancaires. On y observe des transferts et créations d'activités rendus plus aisés depuis les espaces métropolitains (Baudelle et Tallec, 2008), y compris dans des branches caractéristiques de la dématérialisation de l'économie (ingénierie et expertise informatique, édition logicielle). Des villes moyennes continuent à accueillir, dans le Grand Sud-ouest français comme ailleurs, des activités industrielles ou de services avancés plutôt compétitives (Saint-Julien, 2003) même si préexistent des problèmes liés à l'existence locale d'une " masse critique " se rapportant à des marchés de consommation et du travail diversifiés qui garantiraient sur place un seuil de rentabilité économique et marchande à l'offre de services.

Autant pour les " grandes " villes moyennes (supérieures à 100000 habitants avec leur agglomération comme Pau ou la conurbation Bayonne-Biarritz) que pour celles situées non loin d'une capitale régionale (ex. Albi, Castres), la diffusion des services technologiques contribue à l'atténuation des contrastes passés avec les métropoles. Cette évolution est à relier aux dotations plus ou moins récentes des villes moyennes en instituts d'enseignements supérieurs, en centres technologiques de recherche appliquée (Grossetti et Loségo, 2003), alors que l'émergence d'une offre de services avancés semble complète la base économique urbaine de ces villes (Zuliani, 2004 et 2005 ; Tallec et Baudelle, 2008 ; Tallec, 2012). Au-delà, la ville moyenne conserve une primauté forte comme espace de base pour la fourniture de services aux petites et moyennes entreprises ainsi qu'aux institutions locales, publiques et parapubliques. La proximité spatiale devient alors un enjeu crucial de la déconcentration des services spécialisés (conseil, expertise ou ingénierie) au contact de firmes ou d'institutions donneuses d'ordres. L'attachement des PME productrices de services technologiques au territoire n'y est alors que plus marqué, et l'efficience collective attachée à des systèmes locaux d'innovation valorise des mécanismes interactionnels qui se soldent par la consolidation de creusets de compétences en services technologiques (Zuliani, 2005 ; Zuliani et Tallec, 2012).

\subsection{Pour les banques, les assurances et les services informatiques, l'incidence des facteurs sectoriels et locaux}

La couverture des villes moyennes par les banques et assurances, puis les formes territorialisées du développement des services informatiques s'expliquent également par des processus organisationnels. Soit qu'ils mettent en jeu des logiques d'appareils à la fois fournisseurs de services dans la banque et les assurances, soit qu'ils se réfèrent à des formes localisées d'organisation en système productif pour les services informatiques et les activités logicielles. 
Les activités bancaires et d'assurances couvrent l'aire marchande des villes moyennes dans le cadre d'une organisation en réseaux d'établissements : elles s'adressent autant aux particuliers qu'aux professionnels, même s'ils doivent segmenter leur organisation par la création d'entités spécialisées sur chacun de ces deux marchés. La question de la marge de manouvre des services bancaires et d'assurance dans les villes moyennes est intéressante : de quelle autonomie locale disposent ces offreurs et comment s'adaptent-ils aux marchés de ces villes? La démarche analytique privilégie la prise en compte des formes d'organisation adoptées par les fournisseurs de services bancaires et d'assurances pour montrer comment l'échelle géographique de la "ville moyenne " est intégrée dans leur déploiement territorial. D’autant que leurs cadres d'action régionaux revêtent des périmètres spécifiques qui ne recoupent que partiellement les limites des régions administratives traditionnelles. Un principe clé est que selon la nature et la culture des organisations dans la banque et les assurances, les fonctions dévolues aux villes moyennes s'avèrent différentes. En ce sens, on distinguera les structures mutualistes des organisations proprement commerciales. S'il semble que les premières (Crédit Agricole, Banque Populaire ; Mutuelles d'Assurances MACIF ou Groupama) évoluent selon une autonomie gestionnaire attribuée aux établissements locaux, les secondes (dans la banque, BNP, Société Générale...etc ; dans les assurances, Axa, Generali, Aviva...etc) révèlent une structure hiérarchisée insérant les villes moyennes dans des organisations pilotées depuis des centres métropolitains.

A l'opposé, l'essor des services informatiques dans les villes moyennes renvoie à l'émergence de systèmes d'innovation, marquée par l'apparition d'un marché local du travail d'ingénieurs, des stratégies collaboratives entre firmes innovantes, des relations établies par des centres de recherche avec des ingénieristes, et enfin, le rôle clé de personnes-ressources dans les sentiers de croissance. Les villes moyennes révèleraient à leur tour une dynamique vertueuse d'innovation, fondée sur des mécanismes interactionnels propices à la production puis à la circulation des connaissances et compétences appliquées à l'ingénierie logicielle. Au-delà, les formes de changement à l'œuvre posent le principe d'une innovation sociale et institutionnelle (Klein, Tremblay et Fontan, 2009). On la caractérisera par l'efficience d'un gouvernement local impliqué dans le soutien à l'innovation aux côtés d'autres acteurs comme les organisations professionnelles, les centres d'enseignement supérieur et de recherche, les institutions privées ou publiques... L'enjeu consiste en l'affirmation d'un projet de développement qui soit fondée sur la structuration d'un système local d'innovation favorable au renforcement des services technologiques et des activités logicielles.

Le développement des deux corpus de services étudiés dans les villes moyennes doit nous garder cependant de tout excès de déterminisme. Des trajectoires distinctes existent entre les villes en fonction de l'existence d'avantages comparatifs propices à l'essor local des services bancaires et des services informatiques. Et les disparités n'en sont que plus renforcées entre les cités, qu'il s'agisse de la concentration locale des fonctions bancaires et d'assurances, ou bien de la construction localisée de systèmes d'innovation. 


\section{Pour les banques et assurances : des stratégies de localisation différenciées selon la nature des firmes}

L'analyse du déploiement des activités bancaires et d'assurance requiert une approche centrée sur les modes organisationnels qui participent à la diffusion des prestations. Plusieurs types d'organisation des services bancaires et d'assurances préexistent tout en étant fonction de la nature commerciale ou mutualiste des établissements. En outre, leurs aires régionales d'action régionale s'avèrent évolutives à mesure que se réorganisent sur le plan capitalistique les groupes bancaires et/ou d'assurances, comme l'illustrent la fusion intervenue en 2010 entre l'entité Caisse d'Épargne et le réseau des Banques Populaires (création de l'entité "BPCE"), ou les rapprochements établis entre les organismes d'assurances MACIF et MAIF. En fonction du rôle des villes moyennes et de leurs relations aux espaces métropolitains, plusieurs organisations territoriales des banques et organismes d'assurances coexistent :

- Les structures en "région commerciale d'achalandage " (Aquitaine et Midi-Pyrénées réunies) promues essentiellement par les banques commerciales (BNP, Crédit Lyonnais, HSBC... etc) et les grandes sociétés d'assurance (AXA, Generali, Allianz... etc).

- Les organisations en " caisse régionale Grand Sud-ouest ", recoupant en partie le périmètre des trois régions administratives qui en sont constitutives, et qu'initient des groupements mutualistes (MACIF ou MAIF).

- Les organisations en caisses " pluri-départementales " comprenant un centre gestionnaire autonome qui se localise dans une ville moyenne à l'exemple des Banques Populaires ou du Crédit Agricole.

\subsection{Des banques pourvoyeuses de centres d'expertise parmi les villes moyennes}

Dans le cadre de leur organisation générale, certains groupes bancaires (BNP, Crédit Lyonnais, Société Générale) ont procédé à des découpages par métiers et marchés à partir de trois grandes entités : la banque de détail pour les professionnels et particuliers ; la banque dite "corporate" destinée aux entreprises artisanales, aux petits commerces et aux PME ; la banque privée destinée à la gestion des grosses fortunes. La séparation entre la banque de détail et la banque "corporate "se fait selon des seuils de chiffres d'affaires : au-delà de $750000 €$ de chiffre d'affaires pour une entreprise, l'intervention incombe en général aux entités " corporate ". Or, ce découpage n'est pas sans conséquence sur la répartition des compétences d'analyse financière parmi les villes moyennes. Car, dans nombre d'entre elles, la représentation des entreprises (essentiellement des PME) avec moins de $750000 €$ de chiffre d'affaires semble plus prépondérante. 
Selon une logique arborescente, une direction régionale comme celle de la BNP assise sur les territoires d'Aquitaine et de Midi-Pyrénées opère un fractionnement opérationnel à partir de "groupes succursales " qui associent plusieurs agences et constituent chacun un centre de profit ${ }^{7}$. Leur découpage territorial respecte une logique administrative classique : ils peuvent englober deux territoires départementaux ou bien être à cheval sur deux départements voire ne concerner qu'une micro-région avec à chaque fois en leur centre une ville moyenne jouant le rôle de tête de pont coordinatrice. Le système des " groupes succursales " propres aux banques commerciales assure une compétence de proximité visant à expertiser à partir d'une ville moyenne l'attribution de prêts aux PME. Dans le cas du Crédit Lyonnais, cela peut aller jusqu'à organiser dans des villes comme Albi (pour l'ouest de Midi-Pyrénées) ou Nîmes (Nord et Est de Languedoc-Roussillon) des plateformes de services pour expertiser les demandes de financement des petites et moyennes entreprises et pour fournir un appui opérationnel (ressources humaines, politique commerciale, formation aux outils informatiques) aux agences bancaires. Si les banques commerciales développent des structures intermédiaires parmi les villes moyennes pour gérer et administrer ses clientèles d'entreprises, elles opposent en revanche une centralisation territoriale des fonctions techniques ou commerciales d'appui, les structures back office, qui s'opère au profit de la région parisienne (activités de centres d'appels ou de développements informatiques), et dans le Grand Sud-ouest, en faveur des métropoles bordelaise ou toulousaine (activités de gestion des réseaux et sécurité informatique).

La déconcentration des tâches engagée par les banques commerciales, qui font office également de banques d'affaires et d'investissement (BNP, Crédit Lyonnais ou même Groupe CIC), s'inscrit en réponse à l'ancrage local et traditionnel des banques mutualistes (Crédit Agricole, Caisse d'Épargne et Banque Populaire). Face à l'implantation ancienne des institutions coopératives bancaires, un établissement comme la BNP a choisi un maillage du territoire infrarégional à partir de quelques villes moyennes : les "groupes succursales " en tant que services de proximité et d'intermédiation gestionnaire en sont des éléments moteurs et fondent une caractéristique organisationnelle en rupture avec d'autres réseaux d'obédience commerciale. Des entités comme la Société Générale ou la banque HSBC fonctionnent avec des pouvoirs d'expertise concentrés dans un centre directionnel toulousain ou bordelais. Ce modèle centralisé se retrouve parmi les grandes compagnies d'assurances (AXA, Generali, Allianz, Aviva) où une tête directionnelle sise dans une métropole régionale commande des réseaux infrarégionaux de front office. Dans ce cadre, les activités installées dans des villes moyennes se limitent souvent à des bureaux commerciaux en charge de prospecter et gérer des clientèles locales. C'est bien différent de la fonction de "centre relais " qui octroie à la ville mo-

7. Ces groupes succursales représentent des sous-régions administratives également appelées " groupes d'exploitation " (la BNP a tissé un réseau de 11 " groupes succursales " pour l'ensemble de sa direction Aquitaine/Midi-Pyrénées entre autres à Pau, Albi, Agen, Bayonne). 
yenne une fonction intermédiaire et d'appui gestionnaire avec l'objectif de structurer des services bancaires selon une approche maillante du territoire interrégional.

\subsection{Des compétences décisionnelles dans les banques et les assurances parmi les villes moyennes}

D'autres appareils prestataires du secteur bancaire et des assurances dessinent des stratégies d'organisation territoriale qui accordent aux villes moyennes deux rôles possibles : soit celui d'un pôle annexe de décision interdépendant du centre métropolitain de commandement ; soit celui de plateforme gestionnaire au service de l'ensemble d'un réseau régional d'établissements. Les prestations développées procèdent d'entités mutualistes et coopératives dont la gouvernance requiert la participation d'administrateurs élus pour le suivi et la gestion des affaires d'une caisse régionale (MACIF, Groupama, Banque Populaire et Caisse d'Épargne par exemple). Ces acteurs représentent des contrepouvoirs actifs aux structures internes en charge de la gestion et du développement de l'institution de service. Leur action est aussi de veiller à la répartition des activités gestionnaires et décisionnelles au profit des villes moyennes dans lesquelles ils exercent leur mandat électif.

La présence dans les villes moyennes d'activités décisionnelles et gestionnaires dans la banque et les assurances peut résulter d'un schéma collectif de décentralisation des compétences. Dans le Grand Sud-ouest français, l'exemple est fourni par la mutuelle nationale MACIF, entité à l'organisation pyramidale, qui a opté dans les années 1970 pour une organisation en 11 régions commerciales qui sont responsables chacune des actions en direction des sociétaires, des achats et de l'animation des réseaux commerciaux. En revanche, les activités stratégiques (conception des produits, gestion financière, stratégie d'alliances) et le coeur informatique du back office demeurent des compétences parisiennes. La régionalisation a abouti à la création de périmètres territoriaux d'action plus ou moins élargis : ils recoupent pour certains le découpage des régions administratives (Centre, Rhône-Alpes) mais peuvent englober également des territoires plus étendus à l'image de l'entité "Sud-ouest Pyrénées ", répartie entre les régions Aquitaine, Midi-Pyrénées plus les départements de l'Aude et les Pyrénées-Orientales dans la région Languedoc-Roussillon. La décentralisation opérée par la MACIF offre comme particularité l'établissement de sièges sociaux régionaux localisés pratiquement tous dans une ville moyenne. Pour la région "Sud-ouest Pyrénées ", l'installation du siège à Agen s'explique par une localisation optimale entre Toulouse et Bordeaux. Cette structure à la fois siège et back office, forte de 250 personnes, est en charge de la gestion comptable des primes et des sinistres plus les services de protection juridique et contentieux. Des recompositions internes sont à l'œuvre depuis une dizaine d'années : la MACIF s'est engagée dans la diversification de ses métiers ainsi que dans la construction d'une structure de groupe. D'où la recherche d'économies d'échelles avec la centrali- 
sation de nouveaux services (ex : système des inter-mutuelles) et le renforcement de certaines directions centrales à l'échelle de son siège niortais (Poitou Charente), ce qui conduit à s'interroger sur la pérennité des pouvoirs gestionnaires et techniques qui s'étaient construits peu à peu dans une ville moyenne "siège régional " comme Agen.

Les sociétés coopératives d'assurances offrent des modèles d'organisation territoriale distincte dans le cadre de leur fonctionnement régional. Un premier type de structures adopte une organisation centralisée à partir d'un siège unique, établi parfois dans une ville moyenne. Elles se rapportent à la catégorie des mutuelles dites sans " plateformes intermédiaires " (voir l'exemple de la MACIF). Un second ensemble révèle une organisation plus complexe, basée sur des infrastructures de front et back office qui se répartissent parmi l'armature régionale des villes moyennes. Ce type d'organisation est propre en particulier à la mutuelle Groupama qui a constitué à la faveur de fusions entre ses caisses locales, une vaste région commerciale ("Groupama d'Oc ") répartie sur 14 départements très distincts au plan démographique, allant de la Creuse aux Pyrénées Atlantiques et des Landes à la Lozère. Le tout est chapeauté par un siège social métropolitain situé dans l'agglomération toulousaine. Le dispositif territorial adopté par Groupama est fondé sur l'organisation de structures " service après-vente ", sortes de centres de gestion spécialisés par types de sinistres et clientèles. Ils sont répartis dans plusieurs villes moyennes avec l'objectif de fourni un appui aux agences commerciales présentes dans l'ensemble de l'aire d'action régionale de Groupama d'Oc. Une proximité à la fois organisée et géographique se noue entre les divers centres gestionnaires et les opérateurs commerciaux. Avec comme particularité d'offrir une répartition des compétences d'appui entre 9 villes moyennes ${ }^{8}$ en plus de Toulouse.

L'émergence d'une entité géographique et commerciale "Groupama d'Oc " en 2003 a permis de conforter en corollaire un siège "régional " unique installé à Toulouse. Il regroupe l'ensemble des fonctions de support (direction informatique et financière, secrétariat général en charge des relations avec les administrateurs élus, directions des ressources humaines, de la communication) qui sont fournies aux entités commerciales éclatées dans les 14 départements de la région commerciale. Un processus subtil veut que les pouvoirs centralisés au niveau toulousain s'articulent à une réorganisation du réseau des "services après-vente " répartis parmi les villes moyennes. L'objectif est alors de rechercher une spécialisation sectorielle par domaine de clientèles et type de sinistres dans chaque centre périphérique. La répartition des compétences gestionnaires entre le siège toulousain et les plateformes établies dans les villes moyennes se retrouve également parmi des banques mutualistes françaises comme le Crédit Mutuel ou la Banque Populaire. Ces formes d'organisation procèdent d'héritages historiques : les organismes mutualistes bien ancrées en région, qu'ils opèrent dans les banques ou les assurances, sont le produit de fusions successives visant à la constitution d'une territoire

8. Aurillac, Tulle, Guéret, Cahors, Auch, Montauban, Rodez, Pau et Albi. 
critique en termes de clientèles. L'actuelle Banque Populaire Occitane, circonscrite au territoire de Midi-Pyrénées, s'est constituée à partir du regroupement de caisses bi-départementales où certaines villes moyennes (Cahors ou Albi) accueillaient une fonction de siège. Sans remettre en cause ces acquis, l'organisation nouvelle a privilégié plus un schéma en réseau de villes des fonctions directionnelles (exploitation, crédit, développement, affaires juridiques) avec Toulouse, Albi et Cahors qu'une centralisation unique des instances décisionnelles au niveau toulousain.

Dès lors, pourquoi ce souci de conserver un rôle complémentaire aux villes moyennes en tant qu'espaces connexes de gestion et voire de décision interdépendant d'un centre métropolitain ? C'est à ce stade qu'interviennent les formes de régulation à l'œuvre entre les administrateurs élus de l'institution mutualiste et les gestionnaires en charge du développement opérationnel des établissements. Pour des raisons autant historiques que sociologiques qui tiennent à des pratiques d'entraide et de solidarité, le système mutualiste des banques et assurances est bien implanté dans les territoires ruraux. Cet ancrage tient en outre à la représentation élue des administrateurs en charge de représenter les intérêts des membres sociétaires acquéreurs de parts sociales dans les organismes coopératifs. De sorte qu'il existe une tension latente entre les enjeux économiques et d'affaires portés par les acteurs opérationnels, et la légitimité sociale tenue par les administrateurs élus en charge d'un travail d'animation auprès des coopérateurs. Les administrateurs élus sont souvent porteurs d'un modèle territorial visant à atténuer la concentration métropolitaine des activités décisionnelles. Des contreparties en termes d'activités de contrôle, de gestion et parfois de direction sont alors garanties aux villes moyennes. Et ce compromis est d'autant mieux appliqué que certaines cités moyennes du Grand Sud-ouest drainent un nombre de sociétaires plus important que la métropole toulousaine ${ }^{9}$ où règne une forte concurrence entre les firmes bancaires et d'assurances. Malgré une tendance à la concentration toulousaine des fonctions de support, les banques et assurances mutualistes conçoivent de l'intérieur un niveau de régulation territoriale qui accorde des corpus d'activités valorisés aux villes moyennes. Dans certains cas, l'autonomie décisionnelle des appareils bancaires mutualistes fonctionne à partir d'un réseau d'établissements répartis entre plusieurs villes moyennes.

\subsection{Dans le cas du Crédit Agricole Nord Midi-Pyrénées, le rôle structurant des villes moyennes en réseau}

La Caisse Nord Midi-Pyrénées du Crédit Agricole a été fondée en 2004 et s'étend sur quatre départements (Tarn, Tarn et Garonne, Lot et Aveyron). Elle procède de la fu-

\footnotetext{
9. À titre d'exemple, les départements de l'Aveyron et de la Lozère, creusets historiques du développement de Groupama dans la Grand Sud-ouest disposaient en 2008 de 77820 sociétaires contre seulement 44 830 pour l'agglomération toulousaine et la Haute-Garonne (Source : Groupama d'Oc, 2010).
} 
sion des deux anciennes caisses bi-départementales : Sud Alliance (Tarn et Tarn et Garonne) et Quercy/Rouergue (Aveyron et Lot) ${ }^{10}$. La stratégie a consisté sous l'impulsion des sociétaires et leurs représentants élus à organiser un réseau directionnel qui associe quatre villes moyennes : Albi, Rodez, Cahors et Montauban. Une répartition équilibrée des compétences gestionnaires et décisionnelles régit leurs relations. Certes, la ville d'Albi forme le barycentre de la caisse en accueillant plusieurs fonctions directionnelles (direction des ressources humaines, direction des entreprises) en plus des services spécialisés dans l'expertise et l'engagement des risques. On y retrouve également des activités de formation interne qui s'ajoutent à la contribution active de la Caisse Nord Midi-Pyrénées au montage d'une licence professionnelle " Assurance, banque, finance " au Centre de Universitaire d'Albi. Le reste des fonctions directionnelles est réparti entre les autres villes moyennes constitutives du réseau de l'établissement bancaire. Si la direction commerciale est partagée entre Albi et Rodez, le chef lieu de l'Aveyron reçoit la direction de l'informatique bancaire ainsi que l'importante agence en charge du marché des collectivités locales. Pour sa part, Montauban accueille la direction administrative alors que le siège de Cahors est en charge de la direction du risque et de celle du marché professionnel agricole.

Même si Albi exerce une position dominante par la concentration de plusieurs fonctions décisionnelles, le souci semble au maintien d'une équité dans la répartition des responsabilités entre les sites. Le système du sociétariat établit des contraintes fortes pour conserver des centres directionnels en contrepoint d'Albi. La gouvernance alors privilégiée consiste à ce que chaque direction présente dans une ville rayonne sur l'ensemble du territoire de la Caisse selon une imbrication constante des responsabilités entre les sites. Signe d'une recherche affirmée d'autonomie, la Caisse Nord Midi-Pyrénées du Crédit Agricole a développé ses compétences informatiques tout en cherchant à renouveler la structure de son sociétariat. Sur le premier point, l'enjeu a consisté filialiser à Rodez en 1982 des services d'infogérance plus tard intégrés au GIE EXA, devenu au plan national le principal prestataire en informatique de gestion pour les caisses du Crédit Agricole. En deuxième lieu, la relance d'une politique du sociétariat, avec l'appui donné à des projets d'intérêt collectif, intervient au moment où le profil social des administrateurs élus (représentants des coopérateurs) connaît des changements avec une représentation croissante des cadres et professions intermédiaires au détriment des agriculteurs.

La gestion par les banques et les organismes d'assurances de leur implantation régionale produit des impacts différenciés parmi les villes moyennes du Grand Sud-ouest. Ces effets ont trait aux compétences variées que les villes moyennes intègrent dans le cadre du déploiement territorial des réseaux d'établissements. Les organismes bancaires ou d'assurances tissent des formes d'organisation territoriale qui confèrent à quelques

10. La Caisse Nord Midi-Pyrénées du Crédit Agricole gère les comptes de quelque 600000 clients et emploie près de 2000 actifs. 
villes moyennes des attributs directionnels et gestionnaires. De sorte que les formes organisationnelles spécifiques aux réseaux bancaires octroient des statuts distincts aux villes moyennes, ce qui renforce en retour la perception de leur hétérogénéité dans l'armature urbaine régionale.

\section{Parmi plusieurs villes moyennes du Grand Sud-ouest, des systèmes d'innovation dans le software}

\subsection{Selon les villes moyennes, des évolutions distinctes des emplois dans les services informatiques}

Au vu de l'évolution des emplois salariés dans les services informatiques ${ }^{11}$ établie à partir des sources fournies par l'UNEDIC, il ressort que dans la majorité des villes moyennes étudiées, les emplois connaissent un essor soutenu bien que moindre en volume par rapport aux services logistiques et transports de marchandises (Figure 1). Durant la période 2001-2007, quelques pôles de croissance dans les services informatiques émergent, qu'il s'agisse de Pau et de Castres, et alors que le taux de croissance annuel moyen des emplois affiche des hausses soutenues dans la conurbation Bayonne/Biarritz, à Nîmes, Rodez ou bien encore Perpignan. Seule la ville de Tarbes enregistre un recul des emplois dans les services informatiques qui n'est pas étranger à la fragilisation de son tissu industriel marquée par l'arrêt des activités de l'Arsenal (GIAT) en 2005, puis la lente reconversion de la construction ferroviaire dans des activités à forte intensité de connaissance (systèmes d'électronique de puissance pour les TGV). En comparaison, les services de logistique et de transports offrent une dimension moins discriminante parmi les villes moyennes : en dépit d'un recul faible des emplois à Tarbes et d'une stagnation dans des villes " portes " d'agglomération métropolitaine (Montauban, Béziers) en attente d'aménagement de nouvelles plateformes d'activités logistiques, les services de transport de fret affichent partout ailleurs une hausse des emplois, notamment parmi les villes moyennes situées le long de corridors de circulation autoroutière (Agen, Pau, Nîmes, Narbonne), dans des secteurs transfrontaliers proches de l'Espagne (BayonneBiarritz, Perpignan) ou nanties d'un tissu industriel dynamique (Pharmaceutique à Castres, production agroalimentaire et industries mécaniques à Rodez).

Entre 1999 et 2009, la démographie des agglomérations de villes moyennes dans le Grand Sud-ouest français enregistre une évolution plutôt soutenue (Figure 3), qui, pour certaines d'entre elles (Perpignan, Narbonne, Agen, Marmande), épouse les taux de crois-

11. Classe générale d'activités constituée pour les besoins de la recherche à partir de l'agrégation de diverses composantes de la NAF 700 jusqu'à 2008 (voir ci-dessus). Les chiffres de l'emploi relevant du fichier "Unistatis " géré et développé par l'UNEDIC. 

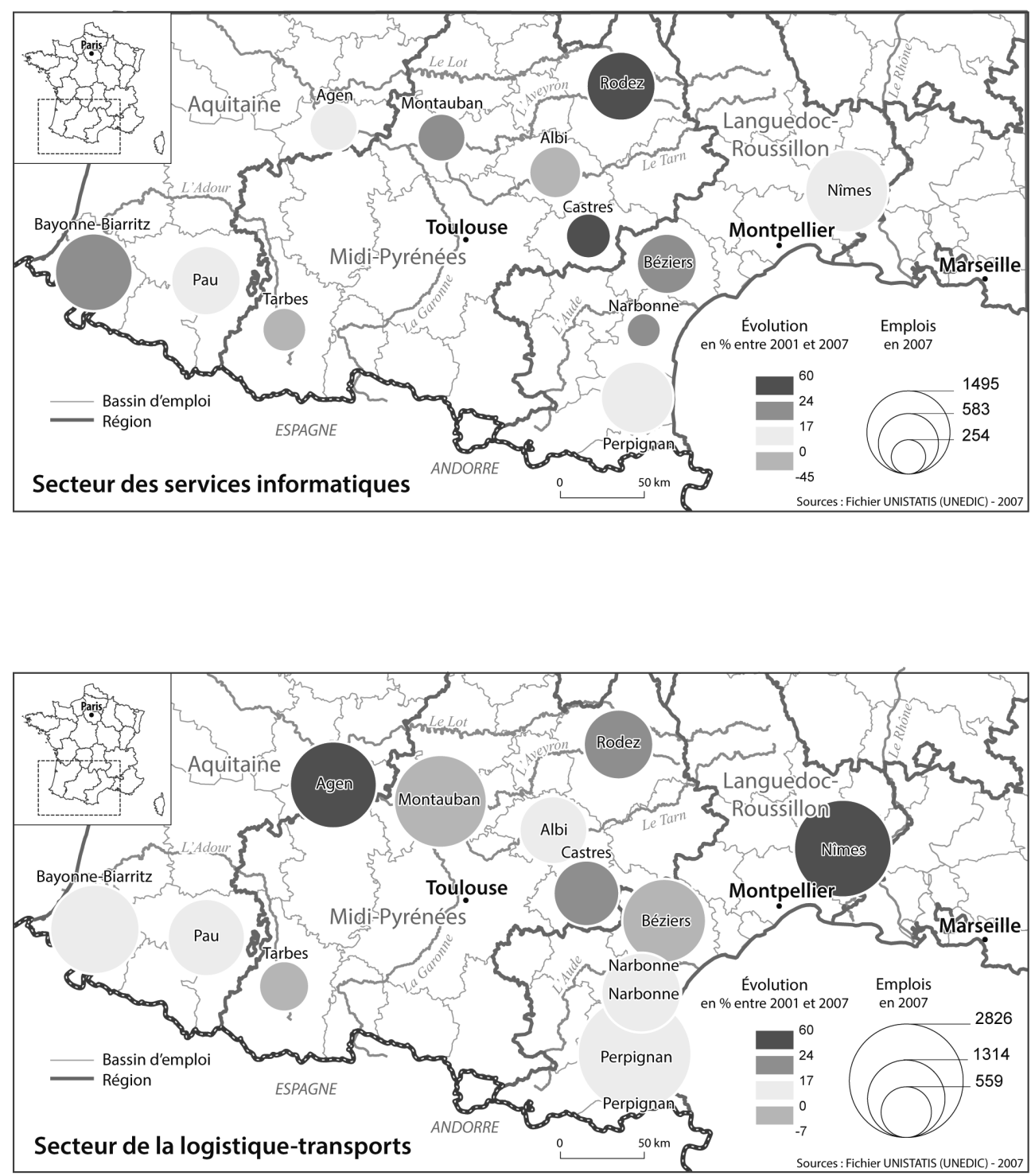

Figure 1. Croissance et répartition des emplois dans les services informatiques et les services de logistique-transports dans 12 villes moyennes du Grand Sud-ouest français (2001-2007).

Zones d'emploi d'Agen, Albi, Bayonne-Biarritz, Béziers, Castres, Montauban, Narbonne, Nîmes, Pau, Perpignan, Rodez et Tarbes. 
sance de Montpellier et Toulouse, deux métropoles françaises connues pour enregistrer les gains de populations parmi les plus élevés. La région Languedoc-Roussillon révèle les plus forts taux de croissance démographique des villes moyennes, notamment pour des agglomérations proches de Montpellier comme Sète et Nîmes. De leur côté, les villes moyennes d'Aquitaine s'inscrivent aussi dans une dynamique démographique forte tant pour les espaces urbains de la vallée de la Garonne (Agen, Marmande) que pour le semis de villes du bassin aturien et de la côte basque. En comparaison, le réseau des villes moyennes de Midi-Pyrénées, à l'exception de Montauban désormais inscrite dans l'aire de croissance toulousaine, offre une armature de villes moins peuplées avec des taux d'accroissement démographique plus faibles. En Languedoc-Roussillon, croissance démographique et affirmation des emplois dans la logistique-transports et les services informatiques se recoupent dans de "grandes " villes moyennes comme Perpignan et Nîmes. A l'inverse, des logiques territoriales de branches déterminent l'essor des emplois dans les services informatiques et la logistique-transports à Rodez et Castres, villes aux gains démographiques modérés. Dans les années 2000, la conjonction entre croissance démographique et hausse des emplois de services semble plus marquée en Aquitaine, notamment dans la conurbation Bayonne-Biarritz pour les prestations informatiques et à Agen dans les services de logistique-transports, ce qui témoigne dans ces villes de contextes territoriaux favorables à l'essor de ces domaines prestataires.

A la fin de la décennie 2000 (chiffres 2008-2010) marquée par l'entrée dans une période de crise, l'évolution des emplois dans les services informatiques en LanguedocRoussillon révèle un recul à peu près général du positionnement des villes moyennes face à la métropole montpelliéraine (Figure 2). Les gains d'emplois attestés depuis 2001 semblent remis en cause sauf à Béziers où l'essor localisé d'une SSII régionale comme Quadria, aux métiers courants dans l'intégration de systèmes mais à la diversification pour l'instant réussie dans la Cloud Computing (services infogérés, hébergement), contribue sur place à une hausse sensible des emplois. En outre, Béziers bien que située dans la catégorie des villes moyennes " médianes " avec quelque 70000 habitants présente un potentiel d'emplois dans les services informatiques pratiquement identique à celui de Nîmes qui se range parmi la catégorie des villes moyennes de taille majeure avec 142000 habitants. Le scénario de la répartition régionale des emplois semble identique pour la branche de la logistique et du transport de marchandises. Face à la concentration continue des emplois à Montpellier, le réseau des villes moyennes subit une érosion de sa position avec une stagnation des emplois dans le pôle pourtant spécialisé en logistique de Perpignan, plus des pertes significatives à Sète ou Carcassonne même si Nîmes de par sa situation sur l'axe de passage entre la vallée du Rhône (Avignon) et Montpellier continue à capter des flux d'activités (ex. installation récente de la base régionale de DHL) dans les transports et l'entreposage. Ces évolutions soulignent pour Languedoc-Roussillon et à la faveur de la crise, une tendance à la restructuration territoriale des deux branches de services avec la poursuite de la concentration métropolitaine des emplois et une tendance secondaire au marquage prestataire des villes moyennes à partir de quelques entreprises bien implantées. 


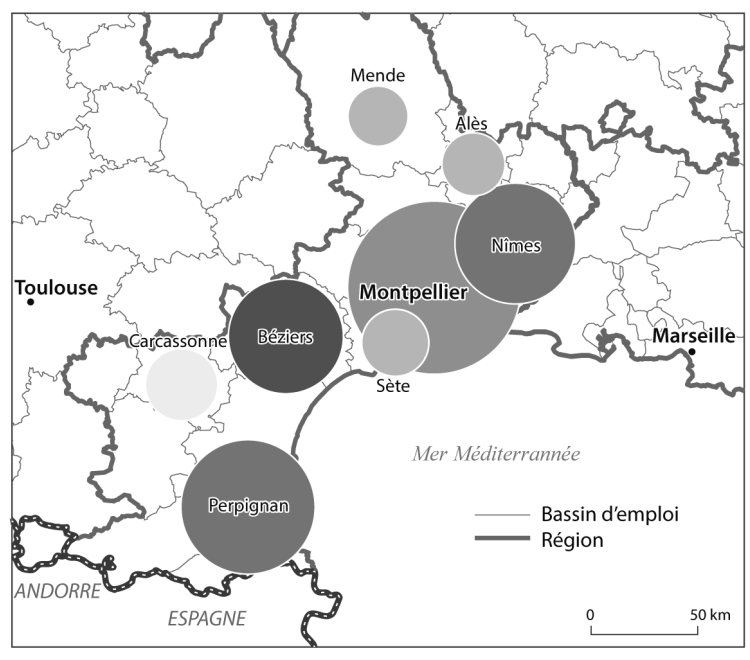

\section{Secteur des services informatiques}

Évolution annuelle moyenne depuis 2008

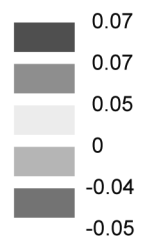

Emplois

en 2010
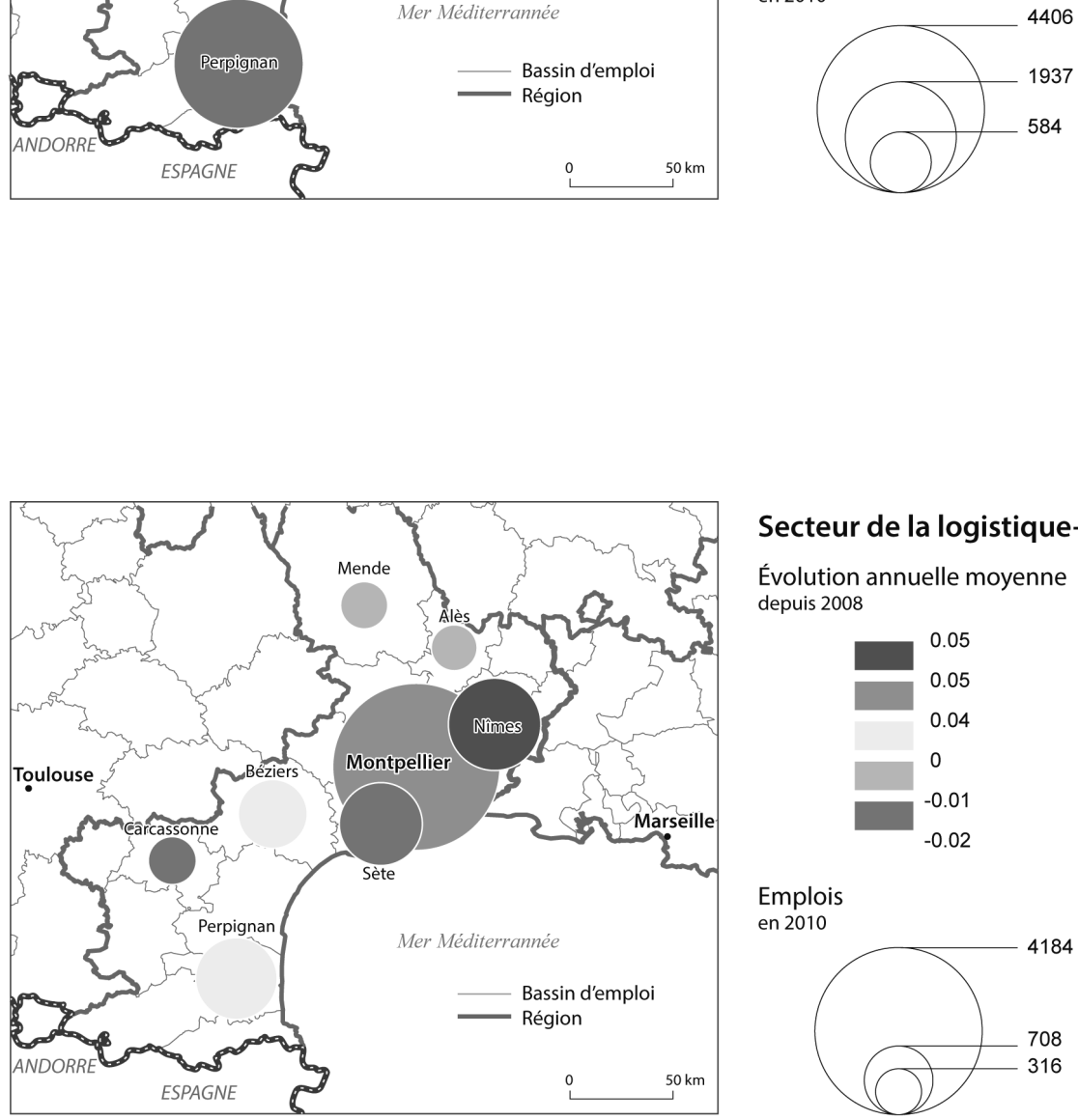

\section{Secteur de la logistique-transports}

Évolution annuelle moyenne depuis 2008

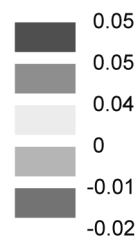

Emplois

en 2010

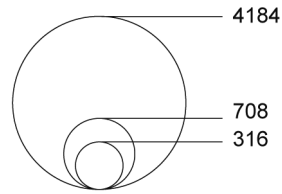

Figure 2. Croissance et répartition des emplois dans les services informatiques et les services de logistique-transports à Montpellier et dans les villes moyennes du Languedoc-Roussillon (2008-2010).

Zones d'emploi de Montpellier, Nîmes, Sète, Mende, Béziers, Carcassonne et Perpignan, 


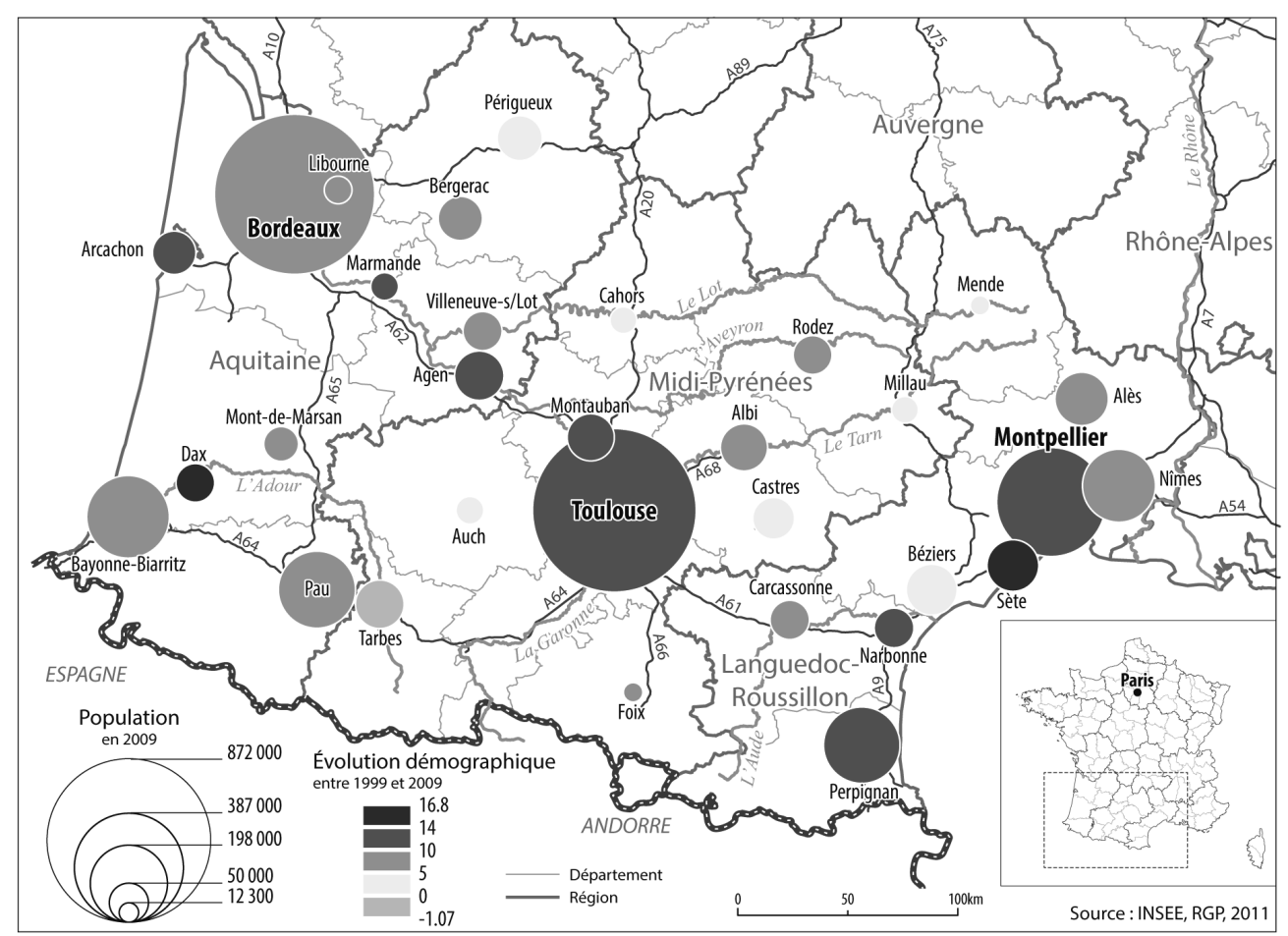

Figure 3. Croissance démographique et distribution de la population dans les principales agglomérations urbaines (définition INSEE) du Grand Sud-ouest français (1999-2009).

\subsection{Des services informatiques inscrits dans les trajectoires économiques des villes moyennes}

L'émergence des services informatiques dans les villes moyennes du Grand Sud-ouest français participe des mutations du contexte économique spécifique à chacune de ces villes. Ces activités prestataires ont pu se structurer grâce à l'affirmation de fonctions de centralité directionnelle ou administrative avec un poids relativement important de l'emploi public et de l'action sociale, ce qui n'est pas sans renforcer la place des villes moyennes dans le réseau urbain régional. Dans d'autres cas, la reconstitution des trajectoires économiques, couplée à celle de l'étude des niveaux d'action et des relations prestataires entre les acteurs, permettent de reconsidérer le rôle dévolu aux villes moyennes comme pôle émergent d'activités de services informatiques (ex. Bayonne/Biarritz, Pau) dans un contexte de développement continu d'activités industrielles ou tertiaires à forte intensité de connaissances. 
Le cas de Bayonne et Biarritz semble représentatif d'un processus territorial par lequel s'est constitué depuis les années 1980 un registre fourni de compétences dans différents champs d'expertise (services informatiques et édition logicielle). Il y a d'abord la genèse de creusets de compétences constituées autour de plusieurs entreprises bénéficiant de la circulation savoir-faire et de personnels diplômés naviguant dans un environnement transfrontalier. Compte-tenu de la diversité des marchés se développant à l'échelle du Pays Basque, trois registres des activités de services se sont affirmés : les services informatiques de type générique (application de gestion pour l'administration, la banque et les assurances), les services d'édition logicielle complétés de fonctions d'ingénierie techniques inscrites dans une proximité de relations avec des établissements industriels (sites de fabrication d'équipements aéronautiques par Dassault à Biarritz ou par Safran-Turboméca à Tarnos, près de Bayonne). Les services informatiques ne sont pas en reste : un pôle de compétences s'est structuré dès les années 80 avec la firme SEI, rachetée en 1992 par le groupe coopératif basque espagnol Mondragón via sa filiale de services informatiques, LKS. SEI va alors développer une offre de solutions dans la gestion de production en travaillant pour la vente par correspondance ainsi que pour divers établissements régionaux du secteur des vêtements et sports de glisse (Quiksilver, Rip Curl, Volcom). Tant l'ancienneté de son implantation que la maîtrise de méthodologies-clés dans la gestion de production et l'informatique industrielle ont conduit à initier un effet diasporique : des personnes issues de l'entreprise ont été à l'initiative de la création de petits établissements innovants (ex. ISEO dans les diagnostics informatisés de la qualité de l'air) à Bayonne et dans d'autres villes proches. D'autres anciens de SEI avaient promu en 1985 un institut du Développement du Logiciel et des Systèmes (IDLS), prédécesseur de l'actuelle école d'ingénieurs ESTIA (École d'Ingénieurs en Technologies Industrielles Avancées), créée sous l'impulsion des décideurs locaux et tournée vers les métiers de l'informatique industrielle. Au-delà, ce sont surtout les spécialités dans l'édition logicielle qui caractérisent le plus le milieu innovant des services informatiques. Diverses petites firmes occupent des niches spécialisées et d'envergure internationale qui requièrent des investissements en recherche et développement : par exemple, la cartographie automatique destinée à la navigation maritime (Maxsea International, 30 emplois) ou le développement de solutions dans la robotique avancée (Robosoft, 22 emplois, firme start-up essaimée de l'INRIA). Et l'échelle géographique diversifiée des clientèles de ces entreprises illustre l'ouverture des creusets d'activités logicielles, dont les marchés dépassent la simple desserte de prestations ou fourniture d'expertise locale.

Au contraire de la conurbation basque, l'espace palois a vu se structurer un tissu industriel constitué d'établissements de grands groupes (Total pour la recherche sur les techniques d'exploration pétrolière et la transformation des hydrocarbures, Safran-Turboméca pour la construction aéronautique, Euralis, pour production de semences agricoles et de denrées agro-alimentaires variées). Or, ces différentes firmes, qui ont en commun de détenir sur place de fortes compétences de recherche et développement sont apparues comme autant d'opportunités pour le développement d'un marché local des 
services informatiques à dominante "technique et industrielle ". On citera en premier lieu l'essor dès les années 1960 de l'activité extractive de gaz naturel à Lacq au Nord de Pau, avec comme prolongement l'implantation en 1985 dans la préfecture du centre scientifique et technique de Total ("Centre Jean Féger "), qui concentre une puissante activité de calculs et simulations. Ces fonctions mobilisent une architecture dédiée en logiciels et systèmes dont une partie est pourvue par de grandes sociétés ingénieristes mais aussi par de petites sociétés fondées par d'anciens ingénieurs provenant de Total ou d'autres établissements locaux de services informatiques. Des interrelations se sont nouées entre les acteurs, contribuant à la formation d'un système d'innovation dans l'informatique scientifique et technique. Cette dynamique est d'autant mieux établie que Pau est le siège de la firme Turboméca (filiale du groupe aéronautique Safran) spécialisée dans la fabrication de moteurs et turbines d'hélicoptères qui emploie sur place 2500 personnes, dont près de 800 exerçant dans les activités de recherche et développement. A l'échelle de l'agglomération paloise, des compétences circulantes affectent la branche des activités logicielles et services liés. Si bien qu'un seuil qualitatif semble avoir été atteint, favorisant même une circulation de compétences entre les entreprises prestataires et firmes spécialisées de logiciels, y compris celles qui œuvrent dans des domaines distincts. En retour, ces mobilités de compétences, et la circulation des savoirs qui lui est consubstantielle confortent une situation assurantielle parmi les offreurs prestataires, quand elles doivent gérer des recrutements spécialisés pour répondre à des marchés extérieurs ou à une demande locale.

Pour sa part, Albi offre le cas d'une ville moyenne considérée comme un territoire de faible tradition industrielle, en quelque sorte le prototype de la ville moyenne dont le développement se serait construit sur une base administrative et la concentration de services "supérieurs " publics. L'industrie n'était pourtant pas en reste en reposant sur l'extraction houillère dans le bassin périphérique de Carmaux, le textile et la verrerie. Au lendemain de la seconde guerre mondiale, le bassin minier d'Albi-Carmaux employait alors près de 7000 actifs. Les difficultés rencontrées par cette activité (rentabilité problématique et coûts de production du charbon jugés élevés dans un contexte de concurrence internationale) ont conduit à son extinction progressive couplée à une restructuration du développement industriel local. Dès lors, un dispositif d'intervention très lourd initié par l'Etat français, les collectivités locales ainsi que la compagnie des Charbonnages de France a visé à une restructuration en profondeur du tissu économique et industriel de l'agglomération albigeoise. Au titre des processus de ré-industrialisation engagés par l'action politique d'Etat figure l'implantation d'une nouvelle école des Mines à Albi-Carmaux, l'EMAC. Or, dès le départ, une des missions centrale de cette école d'ingénieurs généraliste était de faire contribuer par ses activités de recherche appliquée au développement économique local, notamment par l'émergence, le renforcement ou la venue d'entreprises d'ingénierie et d'informatique industrielle. Face aux relations de proximité organisationnelle qui conditionnerait le développement induit des activités d'ingénierie, le faible tissu industriel de l'Albigeois ne semblerait pas réunir les 
attributs du déploiement de ces mêmes activités. Pour autant, l'essor récent de quelques SSII locales tournées vers des marchés nationaux, refond le paysage économique et technologique local. Dans un premier temps, ce saut se manifeste parallèlement à la croissance des emplois de conception-recherche dans l'enseignement supérieur et la recherche (Ecole des Mines, Centre Universitaire J-F Champollion). L'activité industrielle locale ne constitue pas le seul créneau marchand des sociétés albigeoises de services informatiques. Les cellules administratives et de gestion d'activités bancaires et d'assurances ou celles des services aux particuliers intègrent l'utilisation croissante de solutions informatiques, notamment pour l'aide à la décision et à l'organisation.

Au vu de ces caractéristiques structurelles, trois profils peuvent se dégager dans le développement des SSII de l'Albigeois :

- Des sociétés travaillant dans la gestion informatique mais également vers des activités de traitement de données, d'informations et de l'amélioration des procédés industriels. Ce sont donc des applications mais également des prestations que l'on peut qualifier de "génériques " autour de l'ERP, et se déployant autant vers l'industrie que vers les secteurs bancaires, d'assurances et la distribution commerciale ;

- Des sociétés, dont l'activité première réside dans la fourniture de solutions informatiques répondant à des procédés industriels mais pour des marché de niches notamment ceux de l'usinage de précision (ex. Machines-Outils et Conception Assistée par Ordinateur etc...);

- Des jeunes entreprises fournisseuses de prestations de calculs, activités venant consolider leurs carnets de commande. Ces prestations débouchent sur la constitution progressive de solutions informatiques et de logiciels qui leur sont propres.

Le développement d'entreprises fournissant des solutions de Progiciel de Gestion Intégré répond dans un premier temps à une demande locale autour des secteurs bancaires et d'assurances. Sur des bases contractuelles négociées, ces sociétés (Sylob, Report-One ou Inforsud, en tout 200 salariés) développent, pour certaines d'entre-elles, des activités de R\&D en lien étroit avec l'EMAC. Ses spécialités dans le génie des procédés industriels constitue un champ d'enseignement et d'expertise commun partagé avec ces mêmes sociétés (ex. Programme de recherche communs, Thèse CIFRE etc...). Les marchés de Sylob et Report-One sont extérieurs à l'Albigeois faisant évoluer ces sociétés sous le registre d'une concurrence nationale avec d'importants éditeurs de logiciels. L'essentiel des activités de R\&D ou l'édition de ces solutions informatiques produites à Albi sont le fruit d'une division du travail qui s'accompagnent d'activités de prospection disséminées sur l'ensemble du territoire national. Un autre volet du développement local des activités d'ingénierie informatique repose sur des creusets de compétences constituées par des start-up issues de l'incubateur de l'EMAC et créées par des docteurs diplômés de l'Ecole. Leurs activités s'apparentent à l'acquisition progressive d'une fonction d'études appliquées qui vient compléter et diversifier leurs activités d'ori- 
gine. Ces solutions répondent à des niches de marché identifiées par les créateurs, et développées à la suite de programmes de recherche qui amènent ces entreprises à se mouvoir sur des marchés nationaux voire européens animés par de grands groupes industriels (Décathlon, Salomon, Airbus etc...).

Ces exemples de trajectoire économique de villes moyennes, appréhendés à partir de la construction locale de creusets d'activités dans les services informatiques, soulignent que ces agglomérations révèlent chacune des processus structurels spécifiques. Avec comme aboutissement, l'affirmation d'une série d'activités informatiques et innovantes de services aux entreprises ne peut être pour autant analysée sans caractériser une série de causalités interdépendantes à l'échelle de chaque cité.

\subsection{La complémentarité de facteurs " spécifiques » et de facteurs " génériques " constitutive des creusets de services informatiques?}

\subsubsection{Des facteurs " génériques » en lien avec la proximité d'entreprises et/ou d'institutions clientes}

Dans les villes moyennes où les services informatiques restent pourvoyeurs d'emplois, l'analyse par les liens de proximité prestataires confirme une tendance générale à l'accroissement d'une demande locale de travaux en services. Les achats de services peuvent être l'objet d'une sous-traitance ou d'une externalisation des tâches (développement et intégration de systèmes, infogérance, ingénierie diverses et conseils... etc). Sur place, les besoins émanent des donneurs d'ordres du secteur tertiaire, qu'il s'agisse de l'administration et des collectivités territoriales, des entités parapubliques (Directions ou antennes locales d'ERDF, de la SNCF, de La Poste), des entreprises commerciales, ou encore des établissements bancaires détenant une compétence décisionnelle, à l'exemple de la caisse régionale du Crédit Agricole Nord Midi-Pyrénées. Cet opérateur bancaire a étendu son autonomie jusqu'à créer une filiale dédiée au travaux d'informatique de gestion et d'infogérance (Inforsud SA), par la suite cédée en 2004 à la société de services en ingénierie informatique nationale Sopra Group. L'autre foyer demandeur provient des établissements industriels locaux, pour peu qu'ils offrent localement une masse critique d'activités gestionnaires, décisionnelles et technoscientifiques (recherche et technologie), aptes à s'appuyer sur des prestataires externes. La proximité géographique nécessaire à l'accomplissement des prestations s'avère prépondérante et se vérifie dans les " grandes " villes moyennes comme Pau, Bayonne/Biarritz ou encore Nîmes qui cultivent à la fois une densité et une diversité des compétences en matière de services informatiques et d'activités logicielles.

Dans ces villes moyennes supérieures à 100000 habitants ainsi que dans d'autres de moindre importance (Rodez, Albi), mais non dénuées d'une clientèle acheteuse de systèmes logiciels (banques, assurances, centres administratifs voire technico-scientifiques), les volumes croissants de services cédés en sous-traitance ont motivé l'implantation 
d'agences de SSII, majors de la profession en France (Cap Gémini, Atos, Sopra Group). L'arrivée de ces entreprises se produit à partir de certains seuils de marchés qui sont en rapport avec la demande administrative et institutionnelle, quand ce n'est pas avec la demande locale exprimée par des PME et surtout par de grandes firmes industrielles. Ce sont d'abord des équipementiers, soit du secteur aéronautique (Groupe Safran à Pau et Bayonne), soit du secteur automobile (Usine R. Bosch à Rodez), qui entretiennent sur place une capacité combinatoire alliant co-études avec les grands donneurs d'ordres industriels (Airbus, Dassault, Renault, Volkswagen...etc), et cessions de travaux à des ingénieristes locaux. Les besoins d'expertise en conseil et ingénierie informatique proviennent également de grandes entreprises historiquement ancrées dans une ville moyenne qui conservent sur place des activités conceptrices, propices à la venue ou à la création de fonctions connexes d'ingénierie (les Laboratoires Pierre Fabre à Castres, le Centre Technique et Scientifique Jean-Féger, institut central de recherche et développement du groupe pétrolier Total à Pau avec 2300 emplois).

Au-delà même de l'attractivité entretenue par des firmes " locomotives ", la contribution des services informatiques à la production d'externalités territoriales se concrétise par l'émergence de prestations complémentaires. Ce processus se caractérise par la représentation de prestations standardisées, comme le conseil en gestion de production, qui s'adressent à des PME locales ou à des établissements commerciaux. Dans quelques villes porteuses d'une masse critique de services informatiques diversifiés (Pau, Bayonne/Biarritz, Nîmes), des phénomènes d'essaimage en provenance de SSII ou de firmes industrielles conduisent au développement d'applications spécialisées par des petites sociétés, et conviennent aux donneurs d'ordres (informatiques ou industriels) qui y recourent selon une gestion flexible des compétences.

\subsubsection{Des ressources « spécifiques » liées aux formes socio-spatiales de l'innovation}

L'impact des spécialisations industrielles ou technologiques promues par de grandes firmes historiquement implantées dans une ville moyenne constitue un autre déterminant du développement des niches d'activités logicielles. À ce niveau, on soulignera le rôle central exercé par des firmes industrielles qui conservent ou renouvellent des capacités en recherche et technologie ou plus simplement en ingénierie des procédés. Dans le cas du groupe du Groupe Total à Pau, les travaux du groupe pétrolier dédiés aux systèmes SAP ("Systems, applications, and products for data processing ", procédé informatique de gestion de la production) alimentent l'intervention d'une dizaine de consultants très pointus et à même d'élargir leurs activités d'ingénierie et conseil à d'autres secteurs de clientèles, tant sur les marchés nationaux qu'internationaux. Il en est de même à Castres où, dans le secteur pharmaceutique et cosmétique, le Groupe Pierre Fabre (2000 emplois dans le Sud du Tarn) travaille en lien avec des petites firmes partenaires spécialisées et implantées à proximité, au développement et à l'exploitation de technologies dédiées aux évaluations et tests. 
À Tarbes, où depuis le milieu des années 1990 s'opère une reconversion économique axée sur des activités innovantes, Alstom, l'un des principaux établissements industriels de la ville, a engagé une stratégie de rupture à la fois technologique et industrielle. Il a délaissé une activité de fabrication de rames ferroviaires au profit d'une activité de recherche et développement et fabrication dédiée aux systèmes électroniques. Mais cette réorientation s'est accompagnée pour l'établissement Alstom (750 personnes dont 260 ingénieurs et 250 techniciens) d'un investissement actif, en lien avec des collectivités territoriales, dans la création d'un laboratoire de recherche, baptisé PEARL ${ }^{12}$. Cet institut scientifique a pour vocation d'expérimenter et de valider de nouveaux convertisseurs de puissance et systèmes de conversion d'énergie. Rebaptisé "PRIMES ${ }^{13}$ " en 2008, le centre de recherche se fixe comme objectif de développer un pôle public-privé en génie électrique en l'étendant à l'étude de la conversion électronique de puissance et du management des systèmes énergétiques électriques. Des actions collaboratives ont été conçues avec plusieurs centres régionaux de recherche (ENIT, INPT...) ainsi qu'avec diverses sociétés d'ingénierie, dont l'apport de compétences en calculs et tests résulte de l'inauguration d'une plateforme de développement à Tarbes en plus d'une implantation régionale centrale dans la métropolitaine toulousaine. L'enjeu est désormais de faire de PRIMES un outil de recherche à même de développer des technologies et compétences transversales qui irrigue d'autres secteurs industriels régionaux comme l'automobile ou l'aéronautique.

Les villes moyennes, où prévaut l'influence d'une voire deux grandes entreprises industrielles aux activités soutenues de recherche et développement, voient se dessiner une configuration inédite de leurs systèmes d'innovation. C'est le cas à Pau avec la création du Centre de Simulation Palois, sorte de méga-calculateur pour la conception de systèmes informatiques critiques, dont le montage revient à l'équipementier aéronautique Turboméca (Groupe Safran) en lien avec l'antenne locale de la SSII nationale "Communication \& Systèmes ". Ses activités couvrent divers secteurs (aéronautique, automobile, construction ferroviaire... etc) et renvoient à une logique cognitive des travaux de recherche appliquée, transversale à diverses branches industrielles. Dans d'autres situations, la présence de services technologiques rares, axés sur les technologies de l'information et de la communication, s'explique par une insertion dans des organisations industrielles et régionales de type "cluster". Les villes moyennes en constituent ici les points nodaux : Rodez pour l'écosystème industriel de la "Mécanic Vallée " au Sud du Massif Central (Guillaume et Doloreux, 2011) ou bien Bayonne/Biarritz pour le design des vêtements et équipements des sports de glisse (Herrera-Cazenave, 2007). L'enjeu de la proximité y est considéré comme stratégique ne serait-ce que pour mobiliser et fixer une main d'œuvre qualifiée, aménager infrastructures et équipements adaptés (réseaux de communication dans les TIC, instituts de formation supérieure...etc), et attirer d'au-

12. Power Electronics Associated Research Laboratory.

13. Plateform for Research on power electronic Integration and Management of Energy and Storage devices. 
tres entreprises de services partenaires ou sous-traitantes. Ajoutons que dans deux villes où ont été inaugurées des initiatives sectorielles du type "pôle de compétitivité ("Derbi " à Perpignan autour des énergies renouvelables ; "Avenia " à Pau autour des écotechnologies), la finalité des projets est aussi d'aider au développement cumulatif de compétences en test et ingénierie exploitant ou développant, entre autres, des applications logicielles sophistiquées (informatique scientifique et technique).

\subsection{La dynamique territoriale des services informatiques à la base d'une catégorisation des villes moyennes?}

L'ancrage des activités logicielles dans les villes moyennes, soit sur la base des marchés de proximité, soit à partir de spécificités territoriales, conduit à dégager plusieurs catégories de villes moyennes. Des effets de seuils inhérents à la densité des marchés locaux, à une masse critique de ressources endogènes (capital humain, compétences et savoir-faire) et aux relations interactionnelles, se combinent pour expliquer le développement des services informatiques, sans que la taille de la ville ne soit toujours un facteur limitant.

Les " grandes " villes moyennes (Pau, conurbation Biarritz-Bayonne) recèlent des capacités endogènes en faveur du développement des activités logicielles, dont rend compte la taille plus importante des entreprises prestataires ainsi que leurs domaines d'activités diversifiés. En outre, fonctionnent des relations de proximité avec une demande industrielle ou tertiaire versée dans l'innovation de procédés. De là, se sont affirmées des dynamiques cumulatives assez proches de celles à l'œuvre dans les espaces métropolitains : le milieu local des services informatiques lié à des industries innovantes suscite l'attraction d'autres activités qui lui sont reliées et devient une des composantes de la base économique urbaine.

Un autre contexte propice à la polarisation des services informatiques affecte des villes moyennes de taille plus réduite, plutôt confinées aux marges des espaces régionaux (ex. Rodez ou Périgueux). Des creusets de compétences en informatique de gestion ou en gestion de production ont émergé à la faveur des activités promues par des organismes coopératifs (banques, assurances, mutuelles) ou bien d'activités manufacturières restructurées à la suite de crises, quand ce ne sont pas des politiques d'accueil très volontaristes qui ont attiré des éditeurs de logiciels dans des niches de marché. Les fournisseurs locaux de services standardisés (informatique de gestion, ERP) ont développé des liens prestataires vers d'autres activités qui les maintiennent dans le territoire local bien que des contraintes d'origine extérieure conduisent à l'implantation récente de grandes SSII aux palettes de prestations élargies.

Une troisième catégorie de villes moyennes (Albi, Nîmes, Tarbes) offre un profil marqué par un une reconversion industrielle engagée dans les années 1990. Cela se tra- 
duit par l'émergence d'un foyer d'activités dans l'édition logicielle et diverses compétences d'ingénierie. Les conditions mises à ce redéploiement se fondent sur l'existence d'une école d'ingénieurs adossée à un institut de recherche qui inclut parmi ses domaines scientifiques et techniques les technologies logicielles et leurs applications. C'est principalement à partir de ce creuset d'innovations académiques que sont élaborées ou perfectionnées des technologies diffusantes par des petites firmes parfois de création récentes ("start-up»). La proximité d'un espace métropolitain (Toulouse pour Albi ; Montpellier pour Nîmes) ajoute aux attributs territoriaux, dont ces villes moyennes bénéficient sur le plan des débouchés commerciaux, des marchés de l'emploi, et de la circulation de savoirs et des compétences.

À la différence des précédentes, certaines villes moyennes formes des " lieux de polarité " naissants dans les services liés aux TIC mais selon un schéma relationnel étroit avec un espace métropolitain proche. C'est le cas de Castres en Midi-Pyrénées : l'aménagement d'un réseau numérique haut débit lui a permis d'être attractive pour recevoir des fonctions de traitement et gestion informatique alors même que la politique d'externalisation suivie sur place par le groupe pharmaceutique Pierre Fabre aboutissait à agréger sur place une plateforme nationale de services dans l'infogérance. Le choix de privilégier aujourd'hui un développement dans les technologies numériques appliquées à la télémédecine et à l'e-santé se fait dans le cadre de relations renouvelées avec des centres de ressources toulousains (CHU de Toulouse, Centre National d'Études Spatiales) en essayant d'en tirer les avantages pour fixer sur place des projets innovants.

Ces trajectoires de développement n'apparaissent pas pour autant figées tant les mutations organisationnelles des prestataires de services informatiques influent sur les formes d'insertion des villes moyennes dans les systèmes urbains. Nombre de villes moyennes du Grand Sud-ouest s'avèrent des points d'ancrage récent du déploiement des SSII, moyennes ou bien majors de la profession. De telles stratégies sont motivées par le recours accru à l'externalisation de la part de donneurs d'ordres locaux aussi bien de l'industrie que de l'administration (hôpitaux, éducation nationale, fonction préfectorale) et des grands services de consommation (banques, assurances, commerces de détail). De sorte que les besoins renouvelés d'expertises ont déterminé un système de relations plus élaborées de la part des fournisseurs de services : au sein des firmes SSII, les centres de services fonctionnent en réseau par la mobilisation croisée de compétences diverses ou transversales. Et les villes moyennes deviennent de nouveaux maillons territoriaux en lien avec les modalités multiples de diffusion et d'adaptation des compétences prestataires.

À l'inverse, l'édition logicielle participe plutôt à l'exportation de nouvelles richesses compétitives depuis les villes moyennes vers un marché global qui peut être métropolitain, national ou international. Les liens entretenus avec les marchés d'utilisateurs conduisent par des retours d'expérience et en réponse aux besoins, à l'amélioration technologique des produits logiciels. Les modes de distribution spatiale des services, 
qu'ils soient liés aux maillages territoriaux des SSII ou à la capacité exportatrice des éditeurs locaux, insèrent à leur tour les villes moyennes dans des flux globaux où la circulation de méthodologies, compétences et normes concourt au renouvellement local de l'innovation logicielle. Un modèle d'innovation ouvert, nécessaire à la définition puis à la production des systèmes informatiques, s'est donc constitué plutôt dans les villes moyennes où s'exerce une propension au développement cumulatif des services informatiques et activités logicielles.

\section{Conclusion}

Dans les villes moyennes du Grand Sud-ouest, la diversité des situations révélées par la représentation de deux grands registres de services (banques et assurances ; services informatiques et édition logicielle) ne peut plus expliquer de façon plausible qu'il existe d'autres mécanismes pour alimenter ces villes que ceux relatifs à l'influence unique des activités métropolitaines sur le reste de l'espace régional. Le renforcement des activités bancaires et d'assurances ainsi que des services informatiques constitue un indicateur nouveau des recompositions économiques affectant les villes moyennes. La diversité de ces villes tient pourtant à ce que chacune s'est construit un profil particulier qui renvoie entre autres au développement local des activités de services.

Les liens d'intermédiation autonomes avec les espaces métropolitains qu'assument, via les villes moyennes, les banques et assurances, et l'ancrage territorial des services informatiques posent le principe d'un "système de villes moyennes " dans le Grand Sudouest qui agrège plusieurs espaces urbains :

- Les " grandes " villes moyennes (Bayonne/Biarritz, Perpignan et même Pau), lieux de nouvellement actif d'une base d'activités prestataires, où se concrétisent des mécanismes " proto-métropolitains " par une complémentarité naissante de relations entre services technologiques innovants et services divers de support (logistique, maintenance, fonctions d'exécution, centres d'appels...).

- Les villes moyennes " excentrées et isolées " (Rodez, Périgueux) par leur localisation régionale, ce qui conduit certaines d'entre elles à une autonomie par la différenciation locale de l'offre prestataire, non sans accueillir quelques centres décisionnels en réseau de villes dans les secteurs des banques et des assurances mutualistes.

- Les villes moyennes "polarités secondaires fortes" (Albi, Castres, Agen), situées à dans l'orbite des aires métropolitaines mais avec une capacité à fixer des compétences décisionnelles à partir des banques et des assurances mutualistes, alors même que dans certaines d'entre elles s'amorce la construction de systèmes locaux d'innovation pourvus par des activités logicielles. 
- Les villes moyennes touchées par un processus de reconversion économique (Tarbes, Nîmes), également lieux de regroupement des fonctions directionnelles dans la banques coopérative (Crédit Agricole, Banque Populaire), qui sont engagées depuis les années 1990 dans une restructuration industrielle par la recherche de conditions optimales faites au développement d'un système d'innovation dans les technologies logicielles.

Parmi les villes moyennes, le mouvement de tertiarisation, qu'illustrent l'essor des services de consommation (banques et assurances) et la diffusion des activités de services informatiques, ne semble pas achevé. Une variété de situations prévaut selon la spécificité de la demande locale de services, la nature de l'offre prestataire et les liens des firmes de services avec des centres d'activités de niveau supérieur ou des organisations plus globales (firmes multinationales, systèmes de sous-traitance). Par leur déploiement, les services tant bancaires qu'informatiques recomposent l'hétérogénéité structurelle des villes moyennes du Grand Sud-ouest français. Ils révèlent de nouveaux systèmes de villes et l'émergence de creusets d'innovation dans l'ingénierie informatique et l'édition logicielle, même si l'entraînement de ces activités sur les marchés de l'emploi locaux demeure pour l'instant relativement ténu. Dans cette perspective, il y a lieu de réfléchir aux stratégies publiques qui permettraient un maillage renouvelé de l'aire interrégionale du Grand Sud-ouest par les différents profils de villes moyennes ainsi constitués, en tenant compte de leurs capacités intrinsèques d'influence et d'innovation, en plus des complémentarités possibles avec les espaces métropolitains.

\section{Glossaire des sigles}

ASSEDIC : ASSociation pour l'Emploi Dans l'Industrie et le Commerce

BNP : Banque Nationale de Paris

BPCE : Banques Populaires Caisse d'Epargne

CCI : Chambre de Commerce et d'Industrie

CIC : Crédit Industriel et Commercial

CIFRE : Convention Industrielle de Formation par la REcherche

CHU : Centre Hospitalier Universitaire

DATAR : Délégation interministérielle à l'Aménagement du Territoire et à l'Attractivité Régionale

DHL : Dalsay Hillblom Lynn

EMAC : Ecole des Mines d'Albi Carmaux

ENIT : Ecole Nationale d'Ingénieurs de Tarbes

ERDF : Electricité Réseau Distribution France 
ERP : Enterprise Resource Planning

ESTIA : École d'Ingénieurs en Technologies Industrielles Avancées

GIAT : Groupement des Industries de l'Armement Terrestre

GIE : Groupement d'Intérêt Economique

INPT : Institut National Polytechnique de Toulouse

INRIA : Institut National de la Recherche en Informatique et en Automatique

HSBC : Hong Kong \& Shanghai Banking Corporation

MACIF : Mutuelle d'Assurance des Commerçants et Industriels de France et des cadres et des salariés de l'industrie et du commerce

MAIF : Mutuelle d'Assurance des Instituteurs de France

OCDE : Organisation de Coopération et de Développement Economique

SAP : Systems, Applications, and Products for Data Processoring

SNCF : Société Nationale des Chemins de Fer

SSII : Société de Services en Ingénierie Informatique

TGV : Train à Grande Vitesse

TIC : Technologies de l'Information et de la Communication

UNEDIC : Union Nationale interprofessionnelle pour l'Emploi Dans l'Industrie et le Commerce

\section{Bibliographie}

Asheim, B.T. and Gertler, M.S. (2005) " Regional Innovation Systems and the Geographical Foundations of Innovation " in : J. Fagerberg, D. Mowery and R. Nelson (Eds.). Oxford Handbook of Innovation. Oxford University Press, London, pp. 291-317.

Baudelle, G. et Tallec, J. (2008) " Les villes moyennes sont-elles les perdantes de la mondialisation?" Revue Pouvoirs locaux, n 77, 2008, pp. 89-94.

Béhar, D. (2004) “ Les politiques régionales en direction des villes moyennes " in "Les villes moyennes, enjeux pour le développement des territoires ", Acadie/Caisse des Dépôts en Consignations, $30 \mathrm{p}$.
Brunet, R. (1997) «Villes moyennes : point de vue de géographe " in COMMERÇON N. et GOUJON P. (dir) Villes moyennes, espaces sociétés, patrimoine, PUL, pp. 13-25.

Cooke, P., Heidenreich, M. and Braczyk, H-J. (2004) " Regional Innovation Systems : The Role of Governances in a Globalized World ". Routledge, London, 499 p.

Gallouj, C. et alii. (2006) "Services aux entreprises et développement régional : bilan et perspectives ", De Boeck, Bruxelles, 429 p.

Guillaume, R. et Doloreux, D. (2011) “ Production System and Innovation in "Satellite "Regions : lesson from comparaison 
between Mechanic Valley (France) and Beauce (Québec) ». International Journal of Urban and Regional Research, Volume 35.6, pp. 1133-1153.

Grossetti, M. et Losego, P. (dir.) (2003) "La territorialisation de l'enseignement supérieur et de la recherche. France, Espagne, Portugal ", L'Harmattan, Coll. " Géographie en liberté ", 339 p.

Herrera-Cazenave, S. (2007) " La filière glisse en Aquitaine : d'une croissance spontanée à un développement consolidé. L'innovation par la coopération territorialisée " in "Les logiques spatiales de l'innovation sportive. Conditions d'émergence et configuration multiples (sous la direction de Callele J-P. et Menaut, A.), Maison des Sciences de l'Homme d'Aquitaine, pp. 73-93.

Klein, J-L., Fontan, J-M. et Tremblay, D-G. (2009) "Initiatives locales et dynamiques socioterritoriales ", Presse Université du Québec, Montréal, 370 p.

Méndez, R. et alii. (2010) " Estategias de innovación industrial y desarrollo económico en las ciudades intermedias de España ", Ricardo Méndez Eds/Fundación BBVA, Madrid, 605 p.

Mouhoud, E.M. (2010) "Économie de services et développement des territoires ", DATAR-La Documentation Française, Collection " Travaux ", $103 \mathrm{p}$.

Rozenblat, C. et Cicille, P. (2003) " Les villes européennes, analyse comparative ", La Documentation Française/DATAR, Collection "Rapport d'étude ", 94 p.

Saint-Julien, T. (2003) "Les villes moyennes en Europe, contextes et défis ", in Manzagol C., Charbonneau F. et Lewis P. (dir.), Villes moyennes et mondialisation, renouvellement des analyses et des stratégies, Éditions Trames-Montréal, pp. 20-28.

Santamaria, F. (2012) "Les villes moyennes françaises et leur rôle en matière d'amé- nagement du territoire : vers de nouvelles perspectives?", Revue Norois $\mathrm{N}^{\circ} 223$, pp. 13-30.

Tallec, J. (2012) « Les trajectoires économiques et les formes sociales de l'innovation dans les villes moyennes: les cas d'Albi (MidiPyrénées), d'Alès (Languedoc-Roussillon), de Fougères et de Quimper (Bretagne), Revue d'Economie Régionale et Urbaine, $\mathrm{N}^{\circ}$ 2, pp. 192-214.

Taulelle, F. (2010) "La France des villes petites et moyennes " in "La France, une géographie urbaine " (Sous la direction de Laurent Cailly et Martin Vanier), Armand Colin, pp. 149-164.

Scott, A. et Zuliani, JM. (2007) "L'industrie de l'informatique à Toulouse : développement, structure et enjeux (avec Allen J. Scott), Revue d'Économie Régionale et Urbaine, $\mathrm{N}^{\circ} 3$, pp. 339-363.

Zuliani, J-M. (2003) " La diffusion des activités de services dans le réseau urbain de MidiPyrénées : un effet des logiques de branches? ", Revue Sud-ouest Européen No 15 , pp. 67-76.

Zuliani, J-M. (2004) "L'organisation des services aux entreprises dans les villes moyennes du Grand Sud-Ouest français, entre logiques gravitaires et maillages interurbains ", Revue " Géocarrefour " Vol. 79 $\mathrm{N}^{\circ}$ 2, pp. 183-192.

Zuliani, J-M. (2005) "La diffusion des services aux entreprises dans les villes moyennes du Grand Sud-Ouest français ", Revue "Pouvoirs Locaux " N 66, pp. 121-127.

Zuliani, J-M. et Tallec, J. (2012) "Villes moyennes et développement des services technologiques aux entreprises, l'exemple de quatre agglomérations du Grand SudOuest français (Bayonne/Biarritz, Pau, Albi et Castres), Revue " Géographie, Economie, Société ", Volume 14, N 3, pp. 237-259. 
